This item was submitted to Loughborough's Institutional Repository (https://dspace.lboro.ac.uk/) by the author and is made available under the following Creative Commons Licence conditions.

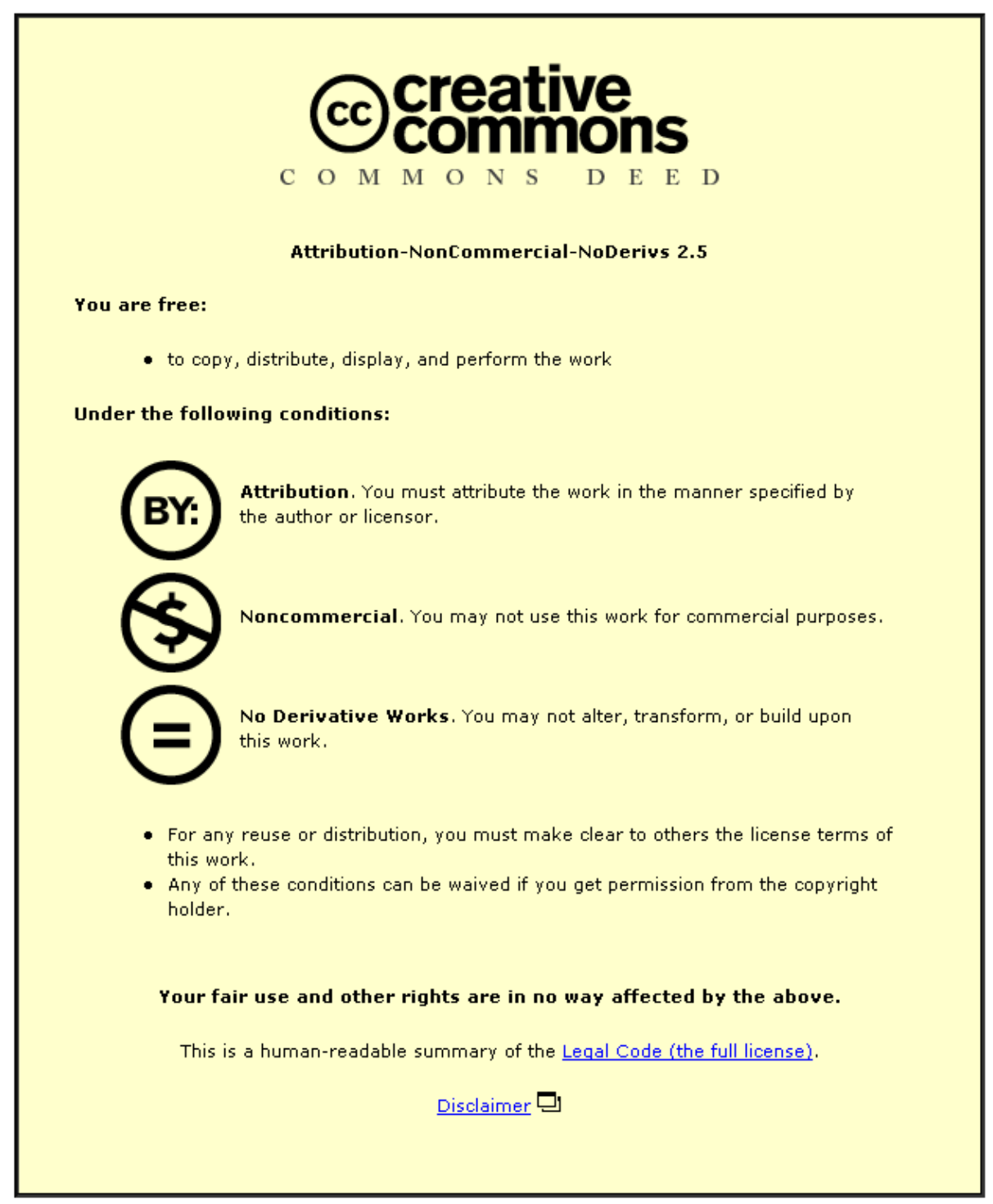

For the full text of this licence, please go to: http://creativecommons.org/licenses/by-nc-nd/2.5/ 


\title{
Freedom and constraint in coupling networks
}

T H Davies

Wolfson School of Mechanical and Manufacturing Engineering, Loughborough University, Loughborough, Leicestershire LE11 3TU, UK

The manuscript was received on 11 April 2005 and was accepted after revision for publication on 9 November 2005.

DOI: $10.1243 / 09544062 C 09105$

\begin{abstract}
Kirchhoff's circulation law has previously been adapted for studies of underconstraint. Here Kirchhoff's cutset law is also adapted for studies of overconstraint. The two procedures are presented in tandem to emphasise their duality. The same tasks are then undertaken using dual virtual power methods. One is a new procedure for underconstraint that introduces the concepts of cutset motion and virtual action, dual with previously reported circuit action and virtual motion. Of the four methods described, the superior ones for each task are identified.
\end{abstract}

Keywords: action, graph, Kirchhoff, motion, screw, virtual power

\section{INTRODUCTION}

In this article, usage of the term coupling is extended to incorporate any means by which actions can be transmitted between bodies. Networks of couplings and the bodies they couple can be underconstrained or overconstrained; simply stiff structures are neither and most useful mechanisms are both. Kirchhoff's circulation law for electrical potential difference, sometimes called the continuity law, is adapted by Davies [1] to provide a robust method for finding all possible motions of which each pair of bodies in underconstrained coupling networks* are capable. It is shown that indirect couplings created by the closure of circuits may suppress some or all of the motions that direct couplings would normally allow. Furthermore, it is shown that the magnitudes of motions that are not suppressed can be expressed in terms of $F_{\mathrm{N}}^{\dagger}$ primary variables, where $F_{\mathrm{N}}$ is the nett degree of freedom (dof) of the coupling network. Tischler and Samuel [3] use the procedure to determine the slop of the finger of a spatial manipulator

\footnotetext{
*Several terms used in this article, coupling and coupling network being examples, are discussed in greater detail by Davies [2]

${ }^{\dagger}$ In reference [1] $F$ is used, whereas here $F$ is the gross dof of a coupling network and is needed as a subscript for vectors and matrices. The distinction between gross and nett dof is explained in section 2.1.
}

caused by clearances in couplings. Tischler [4] uses the procedure to study special configurations of the RCCC linkage. Campos et al. [5] use it in robotics for inverse kinematics and [6] for obstacle detection and avoidance.

There is a related problem concerning overconstrained coupling networks. It is to find the magnitudes of all actions that could be present in terms of $C_{\mathrm{N}}$ primary variables, where $C_{\mathrm{N}}$ is the nett degree of constraint (doc) of the coupling network. Davies [7] uses a virtual power method for this purpose. Fayet $[\mathbf{8}, \mathbf{9}]$ uses vectorial space theory in work that has had similar aims. For assembly tasks, Whitney [10] uses a simpler path method that is applicable to most, but not all, constraint problems.

Here two more methods are introduced that are dual to the two methods $[\mathbf{1}, 7]$ previously reported.

\section{ADAPTATIONS OF KIRCHHOFF'S LAWS}

Two simple examples provide an introduction without involving the topology of general coupling networks.

\subsection{Motions in a single circuit}

All motions that two bodies $i$ and $j$ are capable of belong to a motion screw system of order $f_{i j}$, where 
$f_{i j}$ is said to be the dof of the bodies. If the bodies are uncoupled, $f_{i j}$ is $d$, the dimension* of the screw space. This is the order of the system of all possible screws under consideration. Generally $d$ is six, the value for all screws. Throughout this article, $d$ is six. The motion screw system for two bodies coupled by only one coupling is the $f$-system of the coupling $(f<d)$, where $f$ is the coupling dof. Any motion the coupling can allow is the linear combination of a set of $f$ independent motions that spans the $f$ system. Each motion has an unknown magnitude and $d$ known unit motion coordinates known from the instantaneous geometry.

Consider a kinematic chain of coupled bodies without bifurcations or loops so that, apart from the terminal bodies of the chain, each body is coupled to two other bodies. The motions of two contiguous bodies of the chain are limited only to those of the $f$-system of the coupling between them. When the terminal bodies of the chain are also coupled, a closed circuit of bodies and couplings is created. Now $f_{i j}$ for contiguous bodies $i$ and $j$ may be less than $f$ for their direct coupling because a second coupling exists between those bodies. If there are three or more bodies in the circuit, this second coupling is an indirect coupling between contiguous bodies.

The adaptation of Kirchhoff's circuit law requires that for any closed sequence of bodies ${ }^{\dagger}$ in relative motion, each of the $d$ motion coordinates for contiguous pairs of bodies sum to zero. Thus, for a circuit of couplings and dimension $d$ of six

$$
\Sigma r=\Sigma s=\Sigma t=\Sigma u=\Sigma v=\Sigma w=0
$$

Let the sum of the dof of the couplings within any coupling network be $F$, the gross dof of the coupling network. Each dof corresponds to a motion with an unknown magnitude. Thus, for a coupling network comprising a single circuit of couplings, $d$ equations can be written that express conditions that the $F$ unknowns must satisfy. These equations can be expressed as

$$
\left[\hat{\mathbf{M}}_{\mathbf{D}}\right]_{d, F}[\boldsymbol{\psi}]_{F}=[\mathbf{0}]_{d}
$$

where $\left[\hat{\mathbf{M}}_{\mathbf{D}}\right]_{d, F}$ is the unit motion matrix for direct couplings with elements known from the geometry

\footnotetext{
${ }^{*}$ The only common exception to a dimension of six in kinematics is three for planar kinematics. Using any value other than six, there is a danger of overlooking overconstraint. A dual statement can be made about the dimensions of action systems.

${ }^{\dagger}$ The bodies do not need to be coupled for the adapted law to be valid but the law is of value in this article only when they are.
}

and $[\boldsymbol{\psi}]_{F}$ is the vector of the $F$ unknown magnitudes of angular or translational velocity in the same sequence as the corresponding columns of $\left[\hat{\mathbf{M}}_{\mathbf{D}}\right]_{d, F}$. The solution of equation (2a) identifies which, if any, of the $F$ unknown magnitudes are zero and provides expressions for the remainder in terms of $F_{\mathrm{N}}$ primary variables, where $F_{\mathrm{N}}$ is the nett dof of the coupling network.

A circuit comprising two bodies and two couplings provides an adequate example. Let both be ball and socket couplings called, in Appendix 3 and elsewhere, spheric (S) couplings. Like the $\mathrm{S}$ couplings labelled $\mathbf{A}$ and $\mathbf{B}$ in Fig. 1, a figure illustrating a later example, let there be a frame of reference so that the sphere centres are at $(0,0,0)$ and $(0,0,1)$, respectively.

For an $\mathrm{S}$ coupling, $f=3$. In the absence of another coupling creating a circuit that might further inhibit motions, the motions that an S coupling can permit have (motion) screws confined to a 3-system of screws. Hunt [11] classifies this system as the second special 3-system of screws. The instantaneous screw axes (ISAs) form a bundle of lines through the sphere centre, and all screws have the same pitch. For an S coupling, the pitches are zero, so motions are angular velocities. Each angular velocity is a linear combination of three independent angular velocities having (motion) screws of zero pitch and ISAs along, or parallel to, the frame axes. The unknown magnitudes and known unit motion coordinates for the couplings are listed in the first six rows of Table 3 in Appendix 3. Note that, for coupling $\mathbf{B}$, the rotational velocities parallel to the $x$ - and $y$-axes each cause the point at the origin to have a velocity component.

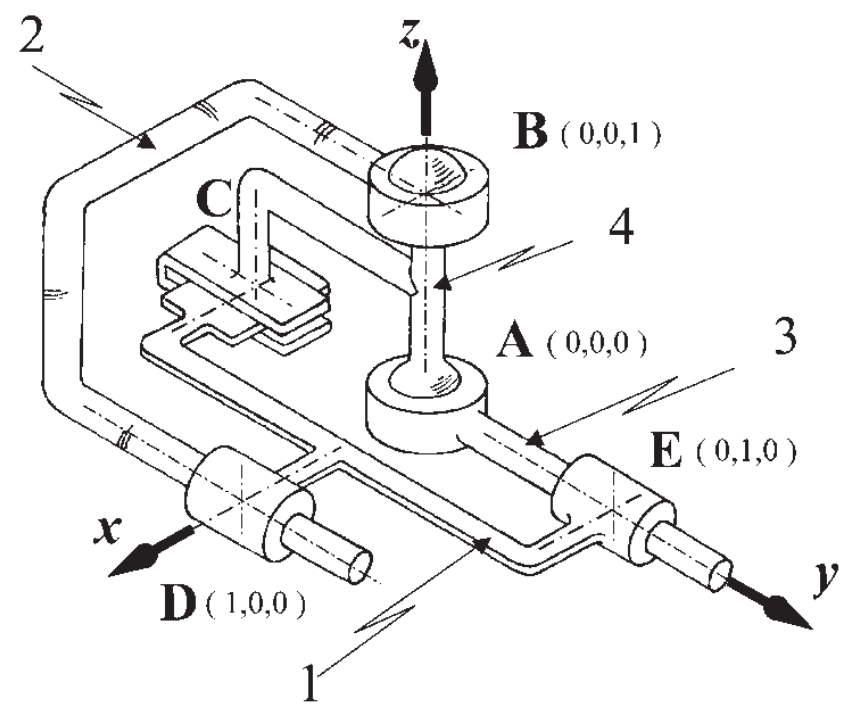

Fig. 1 A spatial coupling network that is both underconstrained and overconstrained 
For the single circuit that has been described, $d=6$ and $F=6$, so equation (2a) becomes

$$
\left[\hat{\mathbf{M}}_{\mathbf{D}}\right]_{6,6}[\boldsymbol{\psi}]_{6}=[\mathbf{0}]_{6}
$$

and thence, by using Appendix 3

$$
\left[\begin{array}{cccccc}
1 & 0 & 0 & 1 & 0 & 0 \\
0 & 1 & 0 & 0 & 1 & 0 \\
0 & 0 & 1 & 0 & 0 & 1 \\
0 & 0 & 0 & 0 & -1 & 0 \\
0 & 0 & 0 & 1 & 0 & 0 \\
0 & 0 & 0 & 0 & 0 & 0
\end{array}\right]_{6,6}\left[\begin{array}{c}
r_{a} \\
s_{a} \\
t_{a} \\
r_{b} \\
s_{b} \\
t_{b}
\end{array}\right]_{6}=\left[\begin{array}{l}
0 \\
0 \\
0 \\
0 \\
0 \\
0
\end{array}\right]_{6}
$$

It is easily seen that all components except $t_{a}$ and $t_{b}$ are zero, and $t_{a}+t_{b}=0$. This means that the only possible motion is rotation about the $z$-axis. $F_{\mathrm{N}}$ is one and so all motions can be expressed in terms of one primary variable, which could be $t_{a}$ or $t_{b}$. Also, $f_{i j}$ has been reduced from three to one. This reduction in freedom is because, by closing a circuit, another coupling exists between the bodies. For this example, with only two bodies, the second coupling is another direct one.

The sixth row of $\left[\hat{\mathbf{M}}_{\mathbf{D}}\right]_{6,6}$, being empty, is redundant. Thus, there are only five independent equations constraining the six components of motion. This indicates that there is overconstraint as well as underconstraint. Overconstraint is studied in the next section.

\subsection{Actions in a single cutset}

For the study of actions in this section, it is helpful to imagine a ring within which an action exists.

Consider a strip of steel; the dimensions are not critical except that, when cold, it can be regarded as stiff. Imagine that the strip is heated until it becomes ductile. Two people each grasp an end of the strip with tongs and then bend, stretch, twist, and generally distort the strip. They bring the ends of the strip together still exerting actions on those ends. The shape does not matter; it could be that of a Möbius strip. A third person now welds the two edges together to create a distorted ring. The ring is then left to cool. Locked into the ring is an action that is a wrench on a screw. The ISA of the wrench will have a fixed location with respect to the ring. The term wrench can be used here because it would be extraordinary if the pitch of the (action) screw happened to be either exactly zero or exactly infinite. As the appendices explain, like motion, action can be quantified by six components. In the absence of any break in the ring, there is no restriction on what these six components could be.
Imagine a spheric (S) coupling with a flange integral with each of the mating elements. The flanges are now bolted to the strip now formed into a ring. The holes in the flanges are guides for a drill so that, when the bolts are inserted and nuts tightened, no change takes place in the action within the ring. Again, let the coupling be labelled $\mathbf{A}$ and let the centre of the sphere be the origin of an orthogonal frame. Next, the original ring is carefully cut between the flanges of the $S$ coupling but without disturbing the coupling or its flanges. The ring remains a single integral body but now with a break bridged by a direct coupling. A small rotation of the S coupling is likely to take place when this cut is made. This rotation is caused by the relief of stresses within the circuit that had been caused by the moment at the origin attributable to the locked-in wrench. Such a moment can no longer exist. For an $S$ coupling, $c=3$. In the absence of another break in the ring that might further inhibit actions, the actions that an $\mathrm{S}$ coupling can transmit have (action) unit screws confined to a 3-system of screws geometrically identical to the (motion) unit screws of the same $S$ coupling. These actions are forces; each is a linear combination of three independent forces having (action) screws of zero pitch with ISAs along the frame axes.

Another S coupling labelled $\mathbf{B}$ is bolted to the ring so that the sphere centre lies at $\left(\begin{array}{lll}0 & 0 & 1\end{array}\right)$. Again, the material of the ring is cut without disturbing the coupling and again a small rotation of this second coupling is likely. To borrow a term from graph theory that is used in its usual context later, a cutset of couplings has been created. A cutset of couplings of a coupling network is a set of couplings the removal of which results in two disjointed subnetworks either or both of which could comprise a single body. The unknown magnitudes and known unit action coordinates for the couplings are listed in the first six rows of Table 4 in Appendix 3. Note that, for coupling $\mathbf{B}$, the forces parallel to the $x$ and $y$-axes each create a moment component at the origin.

The adaptation of Kirchhoff's cutset law requires that for any network of coupled bodies in equilibrium, wherever there exists a cutset of couplings, each of the $d$ action coordinates of those couplings sums to zero. Thus, for $d=6$

$$
\Sigma R=\Sigma S=\Sigma T=\Sigma U=\Sigma V=\Sigma W=0
$$

The single circuit used as an example in the previous section had two couplings but the equation derived was valid for two or more. Dually, the condition stated in equation (1b) is not confined to a cutset of only two couplings. Topologically, a body 
homeomorphic to a sphere with $g$ handles has genus $g$. The ring, a torus, has genus one. Suppose a body of genus $g$ greater than one is prestressed so that there is an action transmitted along each of the $g$ handles and within 'the sphere'.* Suppose also a coupling is bolted to each handle and to 'the sphere', which are then cut in the manner previously described. The coupling network will now comprise two bodies, $l=g$ circuits and one cutset of $e=(l+1)=(g+1)>2$ direct couplings, all coupling the two bodies. This coupling network with more than two couplings is dual with the single circuit with more than two couplings. The cutset comprising only two couplings provides an adequate example however.

Let the sum of the doc of the couplings within any coupling network be $C$, the gross doc of the coupling network. Each doc corresponds to an action with an unknown magnitude. Thus, for a coupling network comprising a single cutset of couplings, $d$ equations can be written that express conditions that the $C$ unknowns must satisfy.

These equations can be expressed as

$$
\left[\hat{\mathbf{A}}_{\mathbf{D}}\right]_{d, C}[\boldsymbol{\Psi}]_{C}=[\mathbf{0}]_{d}
$$

where $\left[\hat{\mathbf{A}}_{\mathbf{D}}\right]_{d, C}$ is the unit action matrix for direct couplings with elements known from the geometry and $[\boldsymbol{\Psi}]_{C}$ is the vector of the $C$ unknown magnitudes of force or torque in the same sequence as the corresponding columns of $\left[\hat{\mathbf{A}}_{\mathbf{D}}\right]_{d, C}$. The solution of equation (2b) identifies which, if any, of the $C$ unknown magnitudes are zero and provides expressions for the remainder in terms of $C_{\mathrm{N}}$ primary variables, where $C_{\mathrm{N}}$ is the nett doc of the coupling network.

For the single cutset that has been described, $d=6$ and $C=6$, so equation (2b) becomes

$$
\left[\hat{\mathbf{A}}_{\mathbf{D}}\right]_{6,6}[\boldsymbol{\Psi}]_{6}=[\mathbf{0}]_{6}
$$

and thence, by using Appendix 3

$$
\left[\begin{array}{cccccc}
0 & 0 & 0 & 0 & -1 & 0 \\
0 & 0 & 0 & 1 & 0 & 0 \\
0 & 0 & 0 & 0 & 0 & 0 \\
1 & 0 & 0 & 1 & 0 & 0 \\
0 & 1 & 0 & 0 & 1 & 0 \\
0 & 0 & 1 & 0 & 0 & 1
\end{array}\right]_{6,6}\left[\begin{array}{c}
U_{a} \\
V_{a} \\
W_{a} \\
U_{b} \\
V_{b} \\
W_{b}
\end{array}\right]_{6}=\left[\begin{array}{l}
0 \\
0 \\
0 \\
0 \\
0 \\
0
\end{array}\right]_{6}
$$

It is easily seen that all components except $W_{a}$ and $W_{b}$ are zero, and $W_{a}+W_{b}=0$. This means that the

\footnotetext{
*The 'sphere' can be any shape provided it has no holes: for example, it could be a distorted flat strip like the strip used to create the ring. The 'handles' can be welded to its ends.
}

only possible action is a force along the $z$-axis. $C_{\mathrm{N}}$ is one and so all motions can be expressed in terms of one primary variable, which could be $W_{a}$ or $W_{b}$. This reduction in constraint is because when the ring is cut a second time, only the second direct coupling remains to close the circuit. Whereas the uncut ring is capable of transmitting all the actions the first coupling can transmit, this may not be true of the second coupling when the ring has been cut.

The third row of $\left[\hat{\mathbf{A}}_{\mathbf{D}}\right]_{6,6}$, being empty, is redundant. Thus, there are only five independent equations constraining the six components of action. This indicates that there is underconstraint as well as overconstraint.

Attention is now paid to coupling networks with both multiple circuits and cutsets.

\subsection{Graphs and matrices of coupling networks}

Chen [12] explains the aspects of graph theory that are required for electrical circuit analysis. They are those required also for the kinestatics of coupling networks with more than one circuit or cutset.

\subsubsection{Coupling graph and its matrices}

The topology of a coupling network can be represented by its coupling graph $G_{C}$ wherein each body is represented by a node and each direct coupling by an edge. A coupling network is shown in Fig. 1. It is referred to hereafter as the 2-circuit network. This is essentially the same as the network devised by Baker [13] and used in reference [1] except that, here in Fig. 1, the ebene (planar) coupling labelled $\mathrm{C}$ is body closed. Body closure enables the coupling to transmit torque having any direction that is perpendicular to the $z$-axis or force along any line parallel to the $z$-axis in either direction. The coupling graph $G_{C}$ of the 2-circuit network is shown in Fig. 2. The four nodes of $G_{C}$ represent the bodies labelled with the corresponding numbers; the five edges represent the couplings labelled with the corresponding letters. A directed graph is needed, so a positive sense is assigned to each edge indicated by an arrow. The choices for the orientations of arrows are arbitrary decisions; for $G_{\mathrm{C}}$, the positive sense assigned to each edge is from the node of lower number towards the node of higher number. The three branches of the chosen tree of $G_{\mathrm{C}}$, edges $a, c$, and $d$, are thick lines; edges $b$ and $e$, the chords, are thinner lines.

Corresponding to the chords are circuits identified by arcs within those circuits. Each circuit is labelled with a letter located within the arc that corresponds to the label of the only edge in the circuit that is a chord. Each circuit is assigned a positive sense indicated by an arrowhead terminating the arc. 


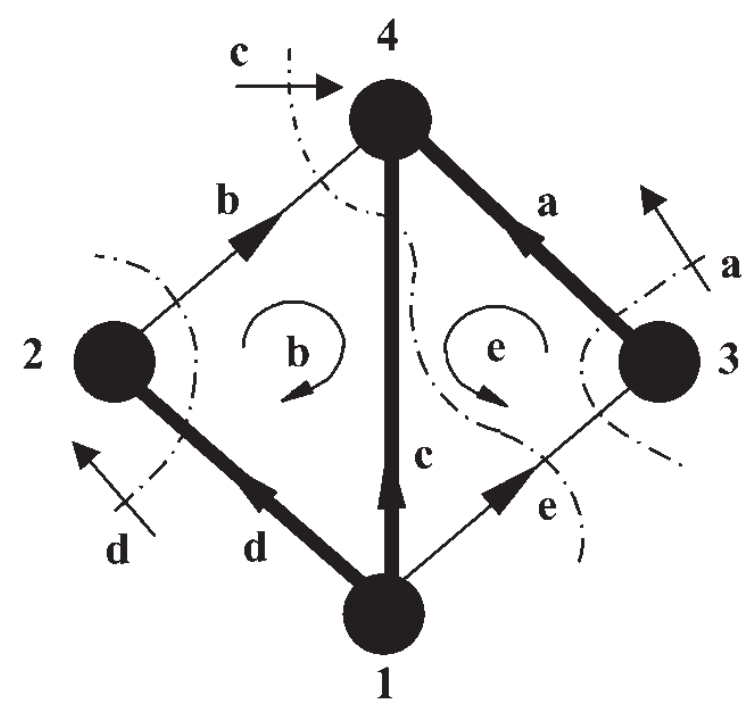

Fig. 2 The coupling graph $G_{C}$ of the coupling network shown in Fig. 1

The positive sense assigned to a circuit is another arbitrary decision. In Fig. 2, the positive sense assigned to each circuit is such that when moving round the circuit in the positive sense given to that circuit, the corresponding chord is traversed in the sense previously assigned to it as being positive.

The removal of the edges of a cutset (of edges) leaves a graph with exactly two components. Each component is a connected subgraph of the original graph. The cutsets corresponding to the branches $a, c$, and $d$ are identified by chain-dashed lines cutting the edges of the cutset. An arrow that identifies the positive sense of the cutset cuts each chaindashed line. Adjacent to each arrow is the label of the cutset corresponding to that of the only edge of the cutset that is a branch. The positive sense assigned to each cutset corresponds to the positive sense of the branch that belongs to it.

The circuit matrix $[\mathbf{B}]_{l, e}$ and the cutset matrix $[\mathbf{Q}]_{k, e}$ of $G_{\mathrm{C}}$ are not required until later. It is important, however, that matrices of the motion graph $G_{\mathrm{M}}$ and the action graph $G_{\mathrm{A}}$, described in subsection 2.4, are compatible with $[\mathbf{B}]_{l, e}$ and $[\mathbf{Q}]_{k, e}$. For $G_{\mathrm{C}}$, the number of circuits $l=2$, the number of cutsets $k=3$, and the number of edges $e=5$. If an edge does not belong to a circuit or cutest, the corresponding element of the matrices is 0 . Other elements are 1 , or -1 , according to whether or not the edge represented has a positive sense that is the same as the positive sense of the circuit or cutset to which it belongs. The circuit and cutset matrices of $G_{\mathrm{C}}$ are as follows

$$
\begin{aligned}
{[\mathbf{B}]_{l, e}=[\mathbf{B}]_{2,5}=} & b \\
e & {\left[\begin{array}{rrrrr}
0 & 1 & -1 & 1 & 0 \\
1 & 0 & -1 & 0 & 1
\end{array}\right] } \\
{[\mathbf{Q}]_{k, e}=[\mathbf{Q}]_{3,5}=} & c\left[\begin{array}{rrrrr}
1 & 0 & 0 & 0 & -1 \\
0 & 1 & 1 & 0 & 1 \\
0 & -1 & 0 & 1 & 0
\end{array}\right]
\end{aligned}
$$

\subsubsection{Motion and action graphs of a coupling network}

In the motion and action graphs now described in tandem, the edges of $G_{\mathrm{C}}$ are replaced by a plurality of edges.

The motion graph $G_{\mathrm{M}}$

In the motion graph $G_{\mathrm{M}}$ of a coupling network, each edge of $G_{C}$ is replaced by $f$ edges in series, where $f$ is the dof of that coupling. A set of $f$ edges in series corresponding to a coupling represents a set of $f$ independent motions that together span the $f$-system of (motion) screws characteristic of the coupling. The circuit matrix of $G_{\mathbf{M}}$ is $\left[\mathbf{B}_{\mathbf{M}}\right]_{l, F}$.

The motion graph $G_{\mathrm{M}}$ of the 2-circuit network is shown in Fig. 3(a). The $f$ values of couplings $a, b$, and $c$ are three and of couplings $d$ and $e$ are two. The four numbered nodes shown as large dots in Fig. 3(a) represent the bodies of the coupling network. The sole function of the remaining nodes, shown as small dots, is to separate the edges incident with them. The choices for the orientation of edges and circuits in $G_{M}$ are the same as those of $G_{C}$ they replace. As in $G_{C}$, these circuits are labelled $b$ and $e$, corresponding to the labels of the corresponding chords of $G_{\mathrm{C}}$ and $G_{\mathrm{M}}$.
The action graph $G_{\mathrm{A}}$

In the action graph $G_{\mathrm{A}}$ of a coupling network, each edge of $G_{\mathrm{C}}$ is replaced by $c$ edges in parallel, where $c$ is the doc of that coupling. A set of $c$ edges in parallel corresponding to a coupling represents a set of $c$ independent actions that together span the $c$-system of (action) screws characteristic of the coupling. The cutset matrix of $G_{\mathrm{M}}$ is $\left[\mathbf{Q}_{\mathbf{A}}\right]_{k, C}$.

The action graph $G_{\mathrm{A}}$ of the 2-circuit network is shown in Fig. 3(b). The $c$ values of couplings $a, b$, and $c$ are three and of couplings $d$ and $e$ are four. The four numbered nodes, shown as pentagons in Fig. 3(b) to avoid congestion caused by multiple edges, represent the bodies of the coupling network. The choices for the orientation of edges and cutsets in $G_{\mathrm{A}}$ are the same as those of $G_{\mathrm{C}}$ they replace. As in $G_{\mathrm{C}}$, these cutsets are labelled $a, c$, and $d$, corresponding to the labels of the corresponding branches of $G_{\mathrm{C}}$ and $G_{\mathrm{A}}$. 
The number of circuits $l$ is 2 ; and the gross network dof $F$ is 13, so the circuit matrix of $G_{\mathrm{M}}$ is

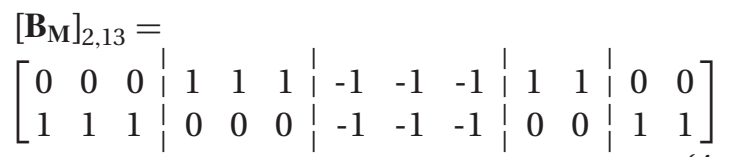

Partition lines are included to emphasize the relationship with $[\mathbf{B}]_{2,5}$.
The number of cutsets $k$ is 3 ; and the gross network $\operatorname{doc} C$ is 17 , so the cutset matrix of $G_{\mathrm{A}}$ is

$\left[\mathbf{Q}_{\mathrm{A}}\right]_{3,17}=$

$\left[\begin{array}{rrr|rrr|rrr|rrrr:rrrr}1 & 1 & 1 & 0 & 0 & 0 & 0 & 0 & 0 & 0 & 0 & 0 & 0 & -1 & -1 & -1 & -1 \\ 0 & 0 & 0 & 1 & 1 & 1 & 1 & 1 & 1 & 0 & 0 & 0 & 0 & 0 & 0 & 0 & 0 \\ 0 & 0 & 0 & -1 & -1 & -1 & 0 & 0 & 0 & 1 & 1 & 1 & 1 & 0 & 0 & 0 & 0\end{array}\right]$

Partition lines are included to emphasize the relationship with $[\mathbf{Q}]_{3,5}$. (a)

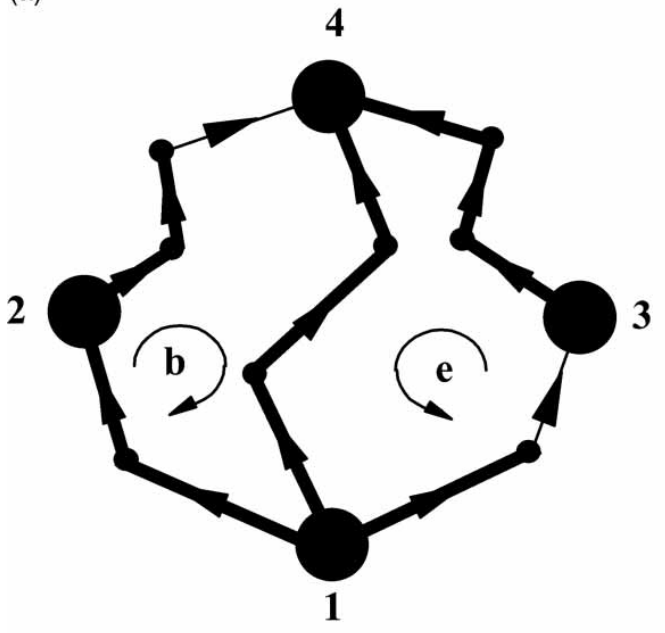

(b)

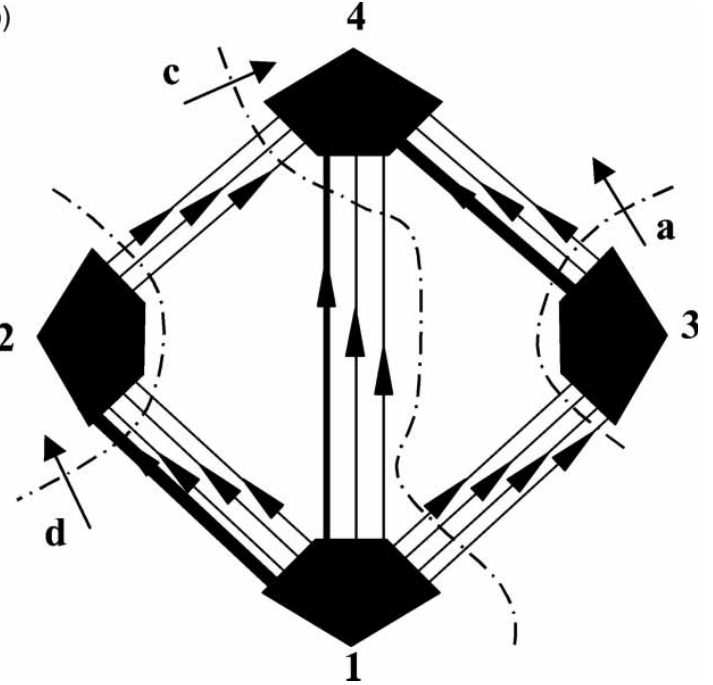

Fig. 3 Two more graphs associated with the coupling network shown in Fig. 1: (a) motion graph $G_{\mathrm{M}}$; (b) action graph $G_{\mathrm{A}}$

\subsection{Matrices of unit motions and actions}

First $\left[\hat{\mathbf{M}}_{\mathbf{D}}\right]_{d, F}$ and $\left[\hat{\mathbf{A}}_{\mathbf{D}}\right]_{d, C}$, the unit motion and action matrices of direct couplings in equations (3a) and (3b), can be assembled.

\subsubsection{Unit matrices of direct couplings}

The elements of these matrices are the subject of Appendix 3.

For the 2-circuit network, the unit motion matrix of direct couplings is

$$
\begin{aligned}
& {\left[\hat{\mathbf{M}}_{\mathbf{D}}\right]_{6,13}=} \\
& {\left[\begin{array}{rrr:rrr:rrr:rr:rr}
1 & 0 & 0 & 1 & 0 & 0 & 0 & 0 & 0 & 0 & 0 & 0 & 0 \\
0 & 1 & 0 & 0 & 1 & 0 & 0 & 0 & 0 & 1 & 0 & 1 & 0 \\
0 & 0 & 1 & 0 & 0 & 1 & 1 & 0 & 0 & 0 & 0 & 0 & 0 \\
0 & 0 & 0 & 0 & -1 & 0 & 0 & 1 & 0 & 0 & 0 & 0 & 0 \\
0 & 0 & 0 & 1 & 0 & 0 & 0 & 0 & 1 & 0 & 1 & 0 & 1 \\
0 & 0 & 0 & 0 & 0 & 0 & 0 & 0 & 0 & 1 & 0 & 0 & 0
\end{array}\right]}
\end{aligned}
$$

For the 2-circuit network, the unit action matrix of direct couplings is

$\left[\hat{\mathbf{A}}_{\mathbf{D}}\right]_{6,17}=$

$\left[\begin{array}{rrr:rrr:lll:lllr:llll}0 & 0 & 0 & 0 & -1 & 0 & 1 & 0 & 0 & 1 & 0 & 0 & 0 & 1 & 0 & 0 & 0 \\ 0 & 0 & 0 & 1 & 0 & 0 & 0 & 1 & 0 & 0 & 0 & 0 & -1 & 0 & 0 & 0 & 0 \\ 0 & 0 & 0 & 0 & 0 & 0 & 0 & 0 & 0 & 0 & 1 & 0 & 0 & 0 & 1 & 0 & 0 \\ 1 & 0 & 0 & 1 & 0 & 0 & 0 & 0 & 0 & 0 & 0 & 1 & 0 & 0 & 0 & 1 & 0 \\ 0 & 1 & 0 & 0 & 1 & 0 & 0 & 0 & 0 & 0 & 0 & 0 & 0 & 0 & 0 & 0 & 0 \\ 0 & 0 & 1 & 0 & 0 & 1 & 0 & 0 & 1 & 0 & 0 & 0 & 1 & 0 & 0 & 0 & 1\end{array}\right]$


The partition lines separate the contributions from the five couplings in the sequence A-E from left to right. For coupling networks with more than one circuit or cutest, these matrices must be adapted.

\subsubsection{Network unit matrices}

There are $d$ equations for each circuit, so for a coupling network having $l$ circuits, there is a total of $d l$ equations. To provide these equations, the known unit motion coordinates are assembled in the network unit motion matrix*

$$
\left[\hat{\mathbf{M}}_{\mathbf{N}}\right]_{d l, F}=\left[\begin{array}{c}
{\left[\hat{\mathbf{M}}_{\mathbf{D}}\right]_{d, F}\left[\mathbf{B}_{1}\right]_{F, F}} \\
{\left[\hat{\mathbf{M}}_{\mathbf{D}}\right]_{d, F}\left[\mathbf{B}_{\mathbf{2}}\right]_{F, F}} \\
\ldots . \\
{\left[\hat{\mathbf{M}}_{\mathbf{D}}\right]_{d, F}\left[\mathbf{B}_{\mathbf{1}}\right]_{F, F}}
\end{array}\right]_{d l, F}
$$

where $\left[\mathbf{B}_{\mathbf{i}}\right]_{F, F}, i=1,2, \ldots, l$, are diagonal matrices with diagonal elements corresponding to row $i$ of $\left[\mathbf{B}_{\mathbf{M}}\right]_{l, F}$. It is essential that the columns of $\left[\hat{\mathbf{M}}_{\mathbf{D}}\right]_{d, F}$ are in the same sequence as the columns of $\left[\mathbf{B}_{\mathbf{M}}\right]_{l, F}$ so that the unit motions screws correspond with the edges of $G_{\mathrm{M}}$ that represent them.

For the 2-circuit network, $d=6, l=2$, and $F=13$, so $\left[\hat{\mathbf{M}}_{\mathbf{N}}\right]_{d l, F}$ becomes

$$
\left[\hat{\mathbf{M}}_{\mathbf{N}}\right]_{12,13}=\left[\begin{array}{l}
{\left[\hat{\mathbf{M}}_{\mathbf{D}}\right]_{6,13}\left[\mathbf{B}_{\mathbf{b}}\right]_{13,13}} \\
{\left[\hat{\mathbf{M}}_{\mathbf{D}}\right]_{6,13}\left[\mathbf{B}_{\mathbf{e}}\right]_{13,13}}
\end{array}\right]_{12,13}
$$

The diagonal matrices, obtained from $\left[\mathbf{B}_{\mathbf{M}}\right]_{2,13}$, are
labelled with the circuit labels $b$ and $e$. For the 2-circuit network

$$
\begin{aligned}
& {\left[\mathbf{B}_{\mathbf{b}}\right]_{13,13}} \\
& \quad=\operatorname{Diag}\left[\begin{array}{lllllllllllll}
0 & 0 & 0 & 1 & 1 & 1 & -1 & -1 & -1 & 1 & 1 & 0 & 0
\end{array}\right]
\end{aligned}
$$

whence

$$
\left[\hat{\mathbf{M}}_{\mathbf{N}}\right]_{12,13}=
$$

$\left[\begin{array}{rrr:rrr:rrr:rr:rr}0 & 0 & 0 & 1 & 0 & 0 & 0 & 0 & 0 & 0 & 0 & 0 & 0 \\ 0 & 0 & 0 & 0 & 1 & 0 & 0 & 0 & 0 & 1 & 0 & 0 & 0 \\ 0 & 0 & 0 & 0 & 0 & 1 & -1 & 0 & 0 & 0 & 0 & 0 & 0 \\ 0 & 0 & 0 & 0 & -1 & 0 & 0 & -1 & 0 & 0 & 0 & 0 & 0 \\ 0 & 0 & 0 & 1 & 0 & 0 & 0 & 0 & -1 & 0 & 1 & 0 & 0 \\ 0 & 0 & 0 & 0 & 0 & 0 & 0 & 0 & 0 & 1 & 0 & 0 & 0 \\ \hdashline & - & - & - & - & - & - & - & - & - & - & - & - \\ 1 & 0 & 0 & 0 & 0 & 0 & 0 & 0 & 0 & 0 & 0 & 0 & 0 \\ 0 & 1 & 0 & 0 & 0 & 0 & 0 & 0 & 0 & 0 & 0 & 1 & 0 \\ 0 & 0 & 1 & 0 & 0 & 0 & -1 & 0 & 0 & 0 & 0 & 0 & 0 \\ 0 & 0 & 0 & 0 & 0 & 0 & 0 & -1 & 0 & 0 & 0 & 0 & 0 \\ 0 & 0 & 0 & 0 & 0 & 0 & 0 & 0 & -1 & 0 & 0 & 0 & 1 \\ 0 & 0 & 0 & 0 & 0 & 0 & 0 & 0 & 0 & 0 & 0 & 0 & 0\end{array}\right]$

There are $d$ equations for each cutest, so for a coupling network having $k$ cutsets, there is a total of $d k$ equations. To provide these equations, the known unit action coordinates are assembled in the network unit action matrix

$$
\left[\hat{\mathbf{A}}_{\mathbf{N}}\right]_{d k, C}=\left[\begin{array}{c}
{\left[\hat{\mathbf{A}}_{\mathbf{D}}\right]_{d, C}\left[\mathbf{Q}_{\mathbf{1}}\right]_{C, C}} \\
{\left[\hat{\mathbf{A}}_{\mathbf{D}}\right]_{d, C}\left[\mathbf{Q}_{\mathbf{2}}\right]_{C, C}} \\
\ldots \\
{\left[\hat{\mathbf{A}}_{\mathbf{D}}\right]_{d, C}\left[\mathbf{Q}_{\mathbf{k}}\right]_{C, C}}
\end{array}\right]_{d k, C}
$$

where $\left[\mathbf{Q}_{\mathbf{i}}\right]_{C, C}, \mathbf{i}=1,2, \ldots, k$, are diagonal matrices with diagonal elements corresponding to row $i$ of $\left[\mathbf{Q}_{\mathbf{A}}\right]_{k, C}$. It is essential that the columns of $\left[\hat{\mathbf{A}}_{\mathbf{D}}\right]_{d, C}$ are in the same sequence as the columns of $\left[\mathbf{Q}_{\mathbf{A}}\right]_{k, C}$ so that the unit actions screws correspond with the edges of $\boldsymbol{G}_{\mathrm{A}}$ that represent them.

For the 2-circuit network, $d=6, k=3$, and $C=17$, so $\left[\hat{\mathbf{A}}_{\mathrm{N}}\right]_{d k, C}$ becomes

$$
\left[\hat{\mathbf{A}}_{\mathbf{N}}\right]_{18,17}=\left[\begin{array}{c}
{\left[\hat{\mathbf{A}}_{\mathbf{D}}\right]_{6,17}\left[\mathbf{Q}_{\mathbf{a}}\right]_{17,17}} \\
{\left[\hat{\mathbf{A}}_{\mathbf{D}}\right]_{6,17}\left[\mathbf{Q}_{\mathbf{c}}\right]_{17,17}} \\
{\left[\hat{\mathbf{A}}_{\mathbf{D}}\right]_{6,17}\left[\mathbf{Q}_{\mathbf{d}}\right]_{17,17}}
\end{array}\right]_{18,17}
$$

The diagonal matrices, obtained from $\left[\mathbf{Q}_{\mathbf{A}}\right]_{3,17}$, are labelled with the cutset labels $a, c$, and $d$. For the 2-circuit network

$\left[\mathbf{Q}_{\mathrm{a}}\right]_{17,17}=\operatorname{Diag}\left[\begin{array}{lllllllllllllllll}1 & 1 & 1 & 0 & 0 & 0 & 0 & 0 & 0 & 0 & 0 & 0 & 0 & -1 & -1 & -1 & -1\end{array}\right]$ whence

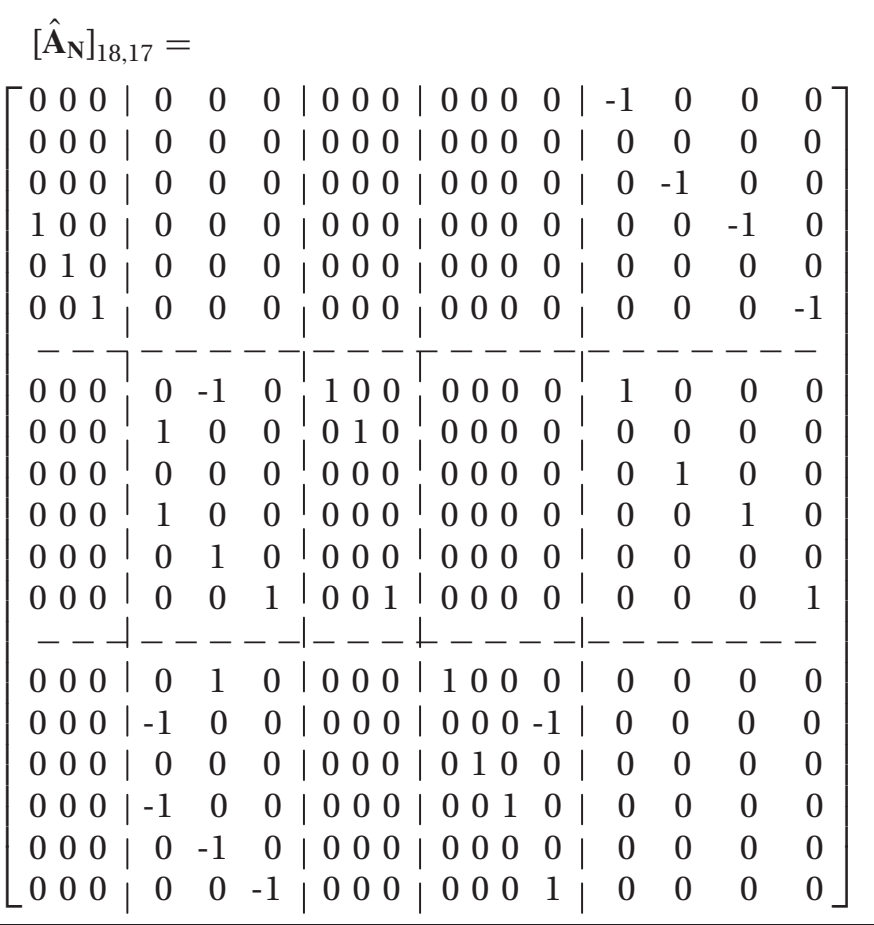

*Previously [1] the procedure for the assembly of this matrix was unclear. 
Lines partition $\left[\hat{\mathbf{M}}_{\mathbf{N}}\right]_{12,13}$ to identify couplings in the sequence $\mathbf{A}-\mathbf{E}$. The row separator divides the matrix into two sets of six rows. Moving downwards, these sets relate to circuits $b$ and $e$, respectively.
Lines partition $\left[\hat{\mathbf{A}}_{\mathbf{N}}\right]_{18,17}$ to identify couplings in the sequence $\mathbf{A}-\mathbf{E}$. The row separators divide the matrix into three sets of six rows. Moving downwards, these sets relate to cutsets $a, c$, and $d$, respectively.

\subsection{Dual equations}

The circuit law requires that when the network unit motion matrix is postmultiplied by $[\boldsymbol{\psi}]_{F}$, the vector of magnitudes of the $F$ motions, the result is a column vector of $d l$ zeros as follows

$$
\left[\hat{\mathbf{M}}_{\mathbf{N}}\right]_{d l, F}[\boldsymbol{\psi}]_{F}=[\mathbf{0}]_{d l}
$$

For the 2-circuit network, the vector $[\boldsymbol{\psi}]_{F}$ of motion magnitudes, listed in Appendix 3, is

$$
[\boldsymbol{\psi}]_{13}=\left[r_{a}, s_{a}, t_{a}, r_{b}, s_{b}, t_{b}, t_{c}, u_{c}, v_{c}, s_{d}, v_{d}, s_{e}, v_{e}\right]^{\mathrm{T}}
$$

The cutset law requires that when the network unit action matrix is postmultiplied by $[\boldsymbol{\Psi}]_{C}$, the vector of magnitudes of the $C$ actions, the result is a column vector of $d k$ zeros as follows

$$
\left[\hat{\mathbf{A}}_{\mathbf{N}}\right]_{d l, C}[\boldsymbol{\Psi}]_{C}=[\mathbf{0}]_{d k}
$$

For the 2-circuit network, the vector $[\boldsymbol{\Psi}]_{C}$ of action magnitudes, listed in Appendix 3, is

$$
\begin{aligned}
{[\boldsymbol{\Psi}]_{17}=} & {\left[U_{a}, V_{a}, W_{a}, U_{b}, V_{b}, W_{b}, R_{c}, S_{c}, W_{c}, R_{d},\right.} \\
& \left.T_{d}, U_{d}, W_{d}, R_{e}, T_{e}, U_{e}, W_{e}\right]^{\mathrm{T}}
\end{aligned}
$$

\subsubsection{Redundant equations}

If there is overconstraint, the rank $m$ of $\left[\hat{\mathbf{M}}_{\mathbf{N}}\right]_{d l, F}$ is less than $d l$, the number of rows, and so

$$
C_{\mathrm{N}}=d l-m
$$

rows are redundant, where $C_{\mathrm{N}}$ is the nett doc.

The rank $m$ of $\left[\hat{\mathbf{M}}_{\mathbf{N}}\right]_{12,13}$ is 10 , and so two rows are redundant and $C_{\mathrm{N}}$ is two. The discarded rows can be the second and the empty twelfth.
If there is underconstraint, the rank $a$ of $\left[\hat{\mathbf{A}}_{\mathbf{N}}\right]_{d k, C}$ is less than $d k$, the number of rows, and so

$F_{\mathrm{N}}=d k-a$

rows are redundant, where $F_{\mathrm{N}}$ is the nett doc.

The rank $a$ of $\left[\hat{\mathbf{A}}_{\mathbf{N}}\right]_{18,17}$ is 15 , and so three rows are redundant and $F_{\mathrm{N}}$ is three. The discarded rows can be the empty second, the third, and the ninth.

\subsubsection{Primary variables}

The remaining $m$ independent equations impose $m$ constraints on the $F$ unknown magnitudes. Thus, these $F$ unknowns can be expressed in terms of $F_{\mathrm{N}}$ primary variables, where

$F_{\mathrm{N}}=F-m$

is the nett dof.

Because the rank $m$ of $\left[\hat{\mathbf{M}}_{\mathbf{N}}\right]_{12,13}$ is 10 the 13 unknowns in $[\boldsymbol{\psi}]_{13}$ are subject to only 10 conditions, so $F_{\mathrm{N}}$ is three, and three independent (primary) variables are needed. The selected primary variables are $s_{a}, t_{a}$, and $v_{c}$.
The remaining $a$ independent equations impose $a$ constraints on the $C$ unknown magnitudes. Thus, these $C$ unknowns can be expressed in terms of $C_{\mathrm{N}}$ primary variables, where

$C_{\mathrm{N}}=C-a$

is the nett doc.

Because the rank $a$ of $\left[\hat{\mathbf{A}}_{\mathbf{N}}\right]_{18,17}$ is 15 the 17 unknowns in $[\boldsymbol{\Psi}]_{17}$ are subject to only 15 conditions, so $C_{\mathrm{N}}$ is two, and two independent (primary) variables are needed. The selected primary variables are $U_{b}$ and $W_{e}$. 


\subsection{Results}

After the solutions of equations (7a) and (7b) are obtained, the vectors of magnitudes $[\boldsymbol{\psi}]_{F}$ and $[\boldsymbol{\Psi}]_{C}$ can be expressed in terms of the primary variables.

In terms of the primary variables $s_{a}, t_{a}$, and $v_{c}$, the vector of all 13 magnitudes

$[\boldsymbol{\psi}]_{13}=\left[0, s_{a}, t_{a}, 0,0, t_{a}, t_{a}, 0, v_{c}, 0, v_{c},-s_{a}, v_{c}\right]^{\mathrm{T}}$
In terms of the primary variables $U_{b}$ and $W_{e}$, the vector of all 17 magnitudes

$$
\begin{array}{r}
{[\boldsymbol{\Psi}]_{17}=\left[-U_{b}, 0, W_{e}, U_{b}, 0,-U_{b}, 0,-U_{b},\right.} \\
\left.W_{c}, 0,0, U_{b},-U_{b}, 0,0,-U_{b}, W_{e}\right]^{\mathrm{T}}
\end{array}
$$

where $W_{c}=\left(U_{b}-W_{e}\right)$. $^{*}$

\subsubsection{Results in matrix form}

A new motion matrix $[\mathbf{M}]_{d, F}$ can now be created by multiplying each column of $\left[\hat{\mathbf{M}}_{\mathbf{D}}\right]_{d, F}$ by the corresponding element of $[\boldsymbol{\psi}]_{F}$ expressed now in terms of the primary variables. Whereas $\left[\mathbf{M}_{\mathbf{D}}\right]_{d, F}$ is the motion matrix for direct couplings expressed in terms of $F$ variables, where $F$ is the gross dof, the new matrix $[\mathbf{M}]_{d, F}$ is expressed in terms of $F_{\mathrm{N}}$ variables.

The unit motion coordinates in $\left[\hat{\mathbf{M}}_{\mathbf{D}}\right]_{6,13}$, when multiplied by the elements of $[\boldsymbol{\psi}]_{13}$, become the matrix of motions

$$
\begin{aligned}
& {[\mathbf{M}]_{6,13}=} \\
& {\left[\begin{array}{ccc:ccc:ccc:cc:cc}
0 & 0 & 0 & 0 & 0 & 0 & 0 & 0 & 0 & 0 & 0 & 0 & 0 \\
0 & s_{a} & 0 & 0 & 0 & 0 & 0 & 0 & 0 & 0 & 0 & -s_{a} & 0 \\
0 & 0 & t_{a} & 0 & 0 & t_{a} & t_{a} & 0 & 0 & 0 & 0 & 0 & 0 \\
0 & 0 & 0 & 0 & 0 & 0 & 0 & 0 & 0 & 0 & 0 & 0 & 0 \\
0 & 0 & 0 & 0 & 0 & 0 & 0 & 0 & v_{c} & 0 & v_{c} & 0 & v_{c} \\
0 & 0 & 0 & 0 & 0 & 0 & 0 & 0 & 0 & 0 & 0 & 0 & 0
\end{array}\right]}
\end{aligned}
$$

A new action matrix $[\mathbf{A}]_{d, C}$ can now be created by multiplying each column of $\left[\hat{\mathbf{A}}_{\mathbf{D}}\right]_{d, C}$ by the corresponding element of $[\boldsymbol{\Psi}]_{C}$ expressed now in terms of the primary variables. Whereas $\left[\mathbf{A}_{\mathbf{D}}\right]_{d, C}$ is the action matrix of direct couplings expressed in terms of $C$ variables, where $C$ is the gross doc, the new matrix $[\mathbf{A}]_{d, C}$ is expressed in terms of $C_{\mathrm{N}}$ variables.

The unit action coordinates in $\left[\hat{\mathbf{A}}_{\mathbf{D}}\right]_{6,17}$, when multiplied by the elements of $[\boldsymbol{\Psi}]_{17}$, become the matrix of actions

$$
\begin{aligned}
& {[\mathbf{A}]_{6,17}=} \\
& {\left[\begin{array}{lll|lll|lll|llll|llll}
0 & 0 & 0 & 0 & 0 & 0 & 0 & 0 & 0 & 0 & 0 & 0 & 0 & 0 & 0 & 0 & 0
\end{array}\right]}
\end{aligned}
$$

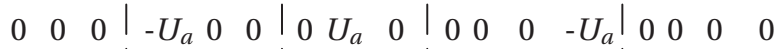

$$
\begin{aligned}
& \begin{array}{lll|lll|lll|llll|llll}
0 & 0 & 0 & 0 & 0 & 0 & 0 & 0 & 0 & 0 & 0 & 0 & 0 & 0 & 0 & 0 & 0
\end{array} \\
& \begin{array}{lll:lll|lll|llll:llll}
U_{a} & 0 & 0 & -U_{a} & 0 & 0 & 0 & 0 & 0 & 0 & 0 & -U_{a} & 0 & 0 & 0 & U_{a} & 0
\end{array} \\
& \begin{array}{lll|lll|lll|llll|llll}
0 & 0 & 0 & 0 & 0 & 0 & 0 & 0 & 0 & 0 & 0 & 0 & 0 & 0 & 0 & 0 & 0
\end{array}
\end{aligned}
$$

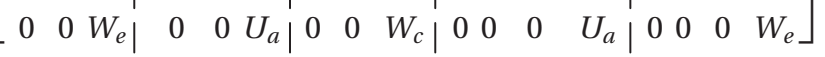

\subsubsection{Contracted matrices}

$[\mathbf{M}]_{d, F}$ can be contracted to $[\mathbf{M}]_{d, e}$ by adding the columns representing the motions associated with each direct coupling. Adding the columns of $[\mathbf{M}]_{6,13}$ that lie between partition lines provides
$[\mathbf{A}]_{d, C}$ can be contracted to $[\mathbf{A}]_{d, e}$ by adding the columns representing the actions associated with each direct coupling. Adding the columns of $[\mathbf{A}]_{6,17}$ that lie between partition lines provides 


$$
[\mathbf{M}]_{6,5}=\left[\begin{array}{ccccc}
0 & 0 & 0 & 0 & 0 \\
s_{a} & 0 & 0 & 0 & -s_{a} \\
t_{a} & t_{a} & t_{a} & 0 & 0 \\
0 & 0 & 0 & 0 & 0 \\
0 & 0 & v_{c} & v_{c} & v_{c} \\
0 & 0 & 0 & 0 & 0
\end{array}\right] \quad[\mathbf{A}]_{6,5}=\left[\begin{array}{ccccc}
0 & 0 & 0 & 0 & 0 \\
0 & U_{b} & -U_{b} & U_{b} & 0 \\
0 & 0 & 0 & 0 & 0 \\
-U_{b} & U_{b} & 0 & U_{b} & -U_{b} \\
0 & 0 & 0 & 0 & 0 \\
W_{e} & -U_{b} & W_{c} & -U_{b} & W_{e}
\end{array}\right]
$$

Each column of $[\mathbf{M}]_{6,5}$ contains the components of the system of motions of which directly coupled bodies could be capable. The sequence of columns corresponds to the direct couplings in alphabetical order A-E.

It is evident from $[\mathbf{M}]_{6,5}$ that $s_{a}$ could be replaced as a primary variable by $-s_{a} ; t_{a}$ by $t_{b}$ or $t_{c}$; and $v_{c}$ by $v_{d}$ or $v_{e}$.
Each column of $[\mathbf{A}]_{6,5}$ contains the components of the system of actions that could be transmitted by the direct couplings. The sequence of columns corresponds to the direct couplings in alphabetical order A-E.

It is evident from $[\mathbf{A}]_{6,5}$ that $U_{b}$ could be replaced as a primary variable by $S_{b},-S_{c}, S_{d},-U_{a}, U_{d},-U_{e}$, - $W_{b}$, or $-W_{d}$; and $W_{e}$ by $W_{a}$ or $W_{c}$.

\subsection{Indirect coupling between bodies}

The original objective of the studies that have led to this article was to find a general and rigorous procedure to determine the dof $f_{i j}$ of indirectly coupled bodies $i$ and $j$ of any kinematic chain. This was achieved and, as explained earlier, in doing so, the components of the motion system of order $f_{i j}$ for those bodies were also obtained [1]. Bodies 2 and 3 of the overconstrained kinematic chain shown in Fig. 1 are indirectly coupled. Once $[\mathbf{M}]_{6,5}$ is found, the final step is briefly stated.

Any path of the coupling graph $G_{\mathrm{C}}$ leading from node 2 to node 3 can be chosen. In $G_{C}$, there are four different paths to choose from, the two simplest comprise only two edges. Consider, as in reference [1], the path comprising edges $b$ and $a$. The components of the motions systems of the compound couplings associated with edges $b$ and $a$ are provided by the second and first columns of $[\mathbf{M}]_{6,5}$, respectively. Note that, when edge $a$ is traversed from node 4 to node 3 , this is the negative sense assigned to that edge. Thus, it is necessary to subtract the first column of $[\mathbf{M}]_{6,5}$ from the second to obtain the components of the motion system for the indirect coupling. The result is

$$
\begin{aligned}
{\left[\mathbf{M}_{23}\right]_{6} } & =\left(0,0, t_{a} ; 0,0,0\right)-\left(0, s_{a}, t_{a} ; 0,0,0\right) \\
& =\left(0,-s_{a}, 0 ; 0,0,0\right)
\end{aligned}
$$

representing angular velocity about the $y$-axis. This result is self-evident from an inspection of Fig. 1.

\section{INTERNAL VIRTUAL POWER METHODS}

Two more methods are now described that perform the same tasks as the adaptations of Kirchhoff's laws. The same examples are also used.

\subsection{Circuit actions and virtual motions in a single circuit}

James Clerk Maxwell is credited with introducing the concept of circuit currents to electrical networks. Because there are always fewer circuits than edges in a graph, information about electrical currents flowing in conductors is more concisely expressed by circuit currents rather than edge currents. Circuit actions are analogous; they provide the same information as coupling actions but do so more concisely.

Locked into the ring described in section 2.2 is a circuit action. It can be called a circuit action because if it were possible to measure stresses in any crosssection of the ring that has been created, it would be found that the resultant action is the same throughout. Nothing is known about this circuit action; it could be any one among the actions that belong to the 6-system of all actions. The circuit action can be represented by the vector $\left[\boldsymbol{A}_{\boldsymbol{l}}\right]_{d}=[R, S, T ; U, V, W]^{\mathrm{T}}$. Normally a subscript is needed to distinguish one circuit from another. Here, this is not necessary because there is only one circuit.

A circuit action cannot expend power on any of the motions that the couplings in the circuit allow when they are unconstrained by circuit closure. Such circuit actions are said to be reciprocal to the $F$ coupling motions spanning the $f$-systems of all the circuit couplings. Thus, $F$ reciprocity equations can be written expressing conditions that the $d$ unknown circuit action components must satisfy. Because the product is zero, unit motion coordinates are sufficient. These are geometric; they are all known and are the elements of $\left[\hat{\mathbf{M}}_{\mathbf{D}}\right]_{d, F}$, the matrix introduced in section 2.1. Here, the transpose of that matrix is needed so that each row contains the $d$ components for one motion. Generally, for a single circuit

$$
\left[\hat{\mathbf{M}}_{\mathbf{D}}^{\mathbf{T}}\right]_{F, d}\left[\boldsymbol{A}_{l}\right]_{d}=[\mathbf{0}]_{F}
$$


Returning to the example described in section 2.2, it is explained that $\mathbf{S}$ couplings are introduced at $\mathbf{A}$ and $\mathbf{B}$. Each coupling has a gross dof of 3 . The two 3 -systems can be spanned by $F=6$ independent motions. Equation (9a) becomes

$$
\begin{aligned}
& {\left[\hat{\mathbf{M}}_{\mathbf{D}}^{\mathbf{T}}\right]_{6,6}\left[\boldsymbol{A}_{l}\right]_{6}=} \\
& {\left[\begin{array}{rrrrrr}
1 & 0 & 0 & 0 & 0 & 0 \\
0 & 1 & 0 & 0 & 0 & 0 \\
0 & 0 & 1 & 0 & 0 & 0 \\
1 & 0 & 0 & 0 & 1 & 0 \\
0 & 1 & 0 & -1 & 0 & 0 \\
0 & 0 & 1 & 0 & 0 & 0
\end{array}\right]_{6,6}\left[\begin{array}{c}
R \\
S \\
T \\
U \\
V
\end{array}\right]_{6}\left[\begin{array}{l}
0 \\
0 \\
0 \\
0 \\
0 \\
0
\end{array}\right]_{6}}
\end{aligned}
$$

It is easily seen that $W$ is indeterminate and the other five components are zero. This means that the only possible circuit action is a force along the $z$-axis.

The third and sixth rows are identical and so one of them is redundant. Thus, there are only five independent equations constraining the six components of circuit action. Again, this indicates that there is underconstraint as well as overconstraint. It means that only the unit angular velocity about the $z$-axis represented by the third and sixth rows of $\left[\hat{\mathbf{M}}_{\mathbf{D}}^{\mathbf{T}}\right]_{6,6}$ could be that of a real angular velocity. All other motions that each coupling would have been capable of allowing in the absence of the second coupling in the circuit are virtual motions. A circuit action cannot expend power on a virtual motion because such a motion cannot exist.

\subsection{Cutset motions and virtual actions in a single cutset}

Dual with the circuit action is the cutset motion. The term cutset was introduced in section 2.2 to describe a set of couplings, the removal of which creates two disconnected subnetworks. For the network described, with only one cutset, the S couplings at A and $\mathbf{B}$ are that cutset and the subnetworks are both single bodies. Before the conditions described subsequently are applied, the motion of the two bodies can be any motion of the cutset motion system represented by the vector $\left[\boldsymbol{M}_{\boldsymbol{k}}\right]_{d}=[r, s, t ; u, v, w]^{\mathrm{T}}$. Normally, these components need a subscript to distinguish one cutset from another. Here, this is not necessary because there is only one cutset.

Generally, for a single cutset, the actions that the couplings of the cutset are capable of transmitting cannot expend power on the cutset motion. The cutset motions are said to be reciprocal to the $c$ coupling actions spanning the $c$-systems of all the couplings, a total of $C$ actions. Thus, $C$ reciprocity equations can be written expressing conditions that the $d$ unknown cutset motion components must satisfy. Because the product is zero, unit action coordinates are sufficient. These are geometric; they are all known and are the elements of $\left[\hat{\mathbf{A}}_{D}\right]_{d, C}$, the matrix introduced in section 2.2. Here, the transpose of that matrix is needed so that each row contains the $d$ components for one action. Generally, for a single cutset

$$
\left[\hat{\mathbf{A}}_{\mathbf{D}}^{\mathbf{T}}\right]_{C, d}\left[\boldsymbol{M}_{\boldsymbol{k}}\right]_{d}=[0]
$$

Returning to the example described in section 2.1, it is explained that $\mathbf{S}$ couplings are introduced at $\mathbf{A}$ and $\mathbf{B}$. Each coupling has a gross doc $c$ of three, so $C$ is six; hence the two 3 -systems can be spanned by six actions. Equation (9b) becomes

$$
\begin{aligned}
& {\left[\hat{\mathbf{A}}_{\mathbf{D}}^{\mathbf{T}}\right]_{6,6}\left[\boldsymbol{M}_{\boldsymbol{k}}\right]_{6}=} \\
& {\left[\begin{array}{rrrrrr}
0 & 0 & 0 & 1 & 0 & 0 \\
0 & 0 & 0 & 0 & 1 & 0 \\
0 & 0 & 0 & 0 & 0 & 1 \\
0 & 1 & 0 & 1 & 0 & 0 \\
-1 & 0 & 0 & 0 & 1 & 0 \\
0 & 0 & 0 & 0 & 0 & 1
\end{array}\right]_{6,6}\left[\begin{array}{c}
r \\
s \\
t \\
u \\
v \\
w
\end{array}\right]_{6}=\left[\begin{array}{l}
0 \\
0 \\
0 \\
0 \\
0 \\
0
\end{array}\right]_{6}}
\end{aligned}
$$

It is easily seen that $t$ is indeterminate and the other five components are zero. This means that the only possible cutset motion is an angular velocity about the $z$-axis.

The third and sixth rows are identical and so one of them is redundant. Thus, there are only five independent equations constraining the six components of cutset motion. Again, this indicates that there is overconstraint as well as underconstraint. It means that only the unit force along the $z$-axis represented by the third and sixth rows of $\left[\hat{\mathbf{A}}_{\mathbf{D}}^{\mathbf{T}}\right]_{6,6}$ could be that of a real force. All other actions that each coupling would have been capable of transmitting in the absence of the second cut in the circuit are virtual actions. Virtual actions cannot exist and therefore cannot expend power on a cutset motion.

\subsection{Cutset motion and circuit action vectors}

Like the adaptations of Kirchhoff's laws, equations must be prepared that are capable of analysing coupling networks that have a plurality of circuits and cutsets. Again, motion is the subject of the left hand column and action in the right hand column. The preparatory work has much in common with section 2: the graphs and network matrices are used again. 
For the general case of a coupling network with $k$ independent cutsets, there are $k$ cutset motion systems each of dimension $d$ and, therefore, a total of $d k$ unknowns. Thus, a total of $C$ equations impose conditions on $d k$ unknown cutset motion components. It is necessary to assemble the $d k$ unknown cutset motion coordinates in a cutset motion vector $\left[\boldsymbol{M}_{\boldsymbol{k}}\right]_{d k}$.

For the 2-circuit network, $\left[\boldsymbol{M}_{\boldsymbol{k}}\right]_{d k}$ becomes $\left[\boldsymbol{M}_{\boldsymbol{k}}\right]_{18}$. In transposed form, this is

$$
\begin{aligned}
{\left[\boldsymbol{M}_{\boldsymbol{k}}\right]_{18}^{\mathrm{T}}=} & {\left[\left[\boldsymbol{M}_{\boldsymbol{a}}\right]_{6}^{\mathrm{T}} \vdots\left[\boldsymbol{M}_{\boldsymbol{c}}\right]_{6}^{\mathrm{T}} \vdots\left[\boldsymbol{M}_{\boldsymbol{d}}\right]_{6}^{\mathrm{T}}\right]_{18}^{\mathrm{T}} } \\
= & {\left[r_{a}, s_{a}, t_{a} ; u_{a}, v_{a}, w_{a} \vdots r_{c}, s_{c}, t_{c} ; u_{c}, v_{c}, w_{c}\right.} \\
& \left.\vdots r_{d}, s_{d}, t_{d} ; u_{d}, v_{d}, w_{d}\right]
\end{aligned}
$$

For the general case of a coupling network with $l$ independent circuits there are $l$ circuit action systems each of dimension $d$ and, therefore, a total of $d l$ unknowns. Thus, a total of $F$ equations impose conditions on $d l$ unknown circuit action components. It is necessary to assemble the $d l$ unknown circuit action coordinates in a circuit action vector $\left[\boldsymbol{A}_{\boldsymbol{l}}\right]_{d l}$.

For the 2-circuit network, $\left[\boldsymbol{A}_{\boldsymbol{l}}\right]_{d l}$ becomes $\left[\boldsymbol{A}_{\boldsymbol{l}}\right]_{12}$. In transposed form, this is

$$
\begin{aligned}
{\left[\boldsymbol{A}_{l}\right]_{12}^{\mathrm{T}}=} & {\left[\left[\boldsymbol{A}_{\boldsymbol{b}}\right]_{6}^{\mathrm{T}} \vdots\left[\boldsymbol{A}_{\boldsymbol{e}}\right]_{6}^{\mathrm{T}}\right]_{12}^{\mathrm{T}} } \\
= & {\left[R_{b}, S_{b}, T_{b} ; U_{b}, V_{b}, W_{b}\right.} \\
& \left.\vdots R_{e}, S_{e}, T_{e} ; U_{e}, V_{e}, W_{e}\right]
\end{aligned}
$$

\subsection{The dual equations}

The unit action matrix $\left[\hat{\mathbf{A}}_{\mathbf{N}}\right]_{d k, C}$ of the coupling network has been derived in section 2.4.2. This matrix contains the $d$ components of the $C$ unit actions in each of the $k$ cutsets. Here, the transpose $\left[\hat{\mathbf{A}}_{\mathbf{N}}^{\mathbf{T}}\right]_{C, d k}$ is needed. To assemble all reciprocity equations, the cutset motion coordinates in the vector $\left[\boldsymbol{M}_{\boldsymbol{k}}\right]_{d k}$ are premultiplied by $\left[\hat{\mathbf{A}}_{\mathbf{N}}^{\mathbf{T}}\right]_{C, d k}$ to give $\left[\hat{\mathbf{A}}_{\mathbf{N}}^{\mathbf{T}}\right]_{C, d k}\left[\boldsymbol{M}_{\boldsymbol{k}}\right]_{d k}=[\mathbf{0}]_{C}$

For the 2-circuit network, $C=17, d=6$, and $k=3$. Equation (11a) can, therefore, be rewritten as $\left[\hat{\mathbf{A}}_{\mathbf{N}}^{\mathbf{T}}\right]_{17,18}\left[\boldsymbol{M}_{\boldsymbol{k}}\right]_{18}=[\mathbf{0}]_{17}$

where

$$
\left[\hat{\mathbf{A}}_{\mathbf{N}}^{\mathbf{T}}\right]_{17,18}=
$$

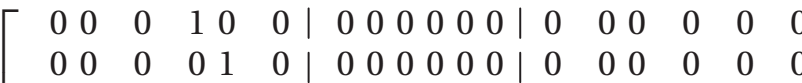

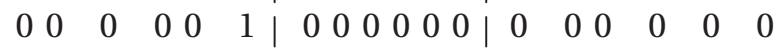

\begin{tabular}{rrrrrr|rrrrrr|rrrrrrr}
0 & 0 & 0 & 0 & 0 & 0 & 0 & 1 & 0 & 1 & 0 & 0 & 0 & -1 & 0 & -1 & 0 & 0
\end{tabular} \begin{tabular}{llllll|llllll|llllll}
0 & 0 & 0 & 0 & 0 & 0 & -1 & 0 & 0 & 0 & 1 & 0 & 1 & 0 & 0 & 0 & -1 & 0
\end{tabular} \begin{tabular}{llllll|llllll|lllllll}
0 & 0 & 0 & 0 & 0 & 0 & 0 & 0 & 0 & 0 & 0 & 1 & 0 & 0 & 0 & 0 & 0 & -1
\end{tabular} \begin{tabular}{llllll|llllll|lllllllllllllllll}
0 & 0 & 0 & 0 & 0 & 0 & 1 & 0 & 0 & 0 & 0 & 0 & 0 & 0 & 0 & 0 & 0 & 0
\end{tabular} \begin{tabular}{llllll|llllll|llllllllllllllll}
0 & 0 & 0 & 0 & 0 & 0 & 0 & 1 & 0 & 0 & 0 & 0 & 0 & 0 & 0 & 0 & 0 & 0
\end{tabular} \begin{tabular}{llllll|llllll|llllll}
0 & 0 & 0 & 0 & 0 & 0 & 0 & 0 & 0 & 0 & 0 & 1 & 0 & 0 & 0 & 0 & 0 & 0
\end{tabular} $--------1-----1-------$ \begin{tabular}{llllll|llllll|lllllll}
0 & 0 & 0 & 0 & 0 & 0 & 0 & 0 & 0 & 0 & 0 & 0 & 1 & 0 & 0 & 0 & 0 & 0
\end{tabular} \begin{tabular}{llllll|llllll|llllll}
0 & 0 & 0 & 0 & 0 & 0 & $\mid$ & 0 & 0 & 0 & 0 & 0 & 0 & 0 & 0 & 1 & 0 & 0
\end{tabular} \begin{tabular}{llllll|llllll|llllll}
0 & 0 & 0 & 0 & 0 & 0 & 0 & 0 & 0 & 0 & 0 & 0 & 0 & 0 & 0 & 0 & 1 & 0
\end{tabular} \begin{tabular}{llllll|llllll|lllllll}
0 & 0 & 0 & 0 & 0 & 0 & 0 & 0 & 0 & 0 & 0 & 0 & 0 & -1 & 0 & 0 & 0 & 1
\end{tabular} $--------1-----1--------$ \begin{tabular}{rlllll|llllll|lllllll}
-1 & 0 & 0 & 0 & 0 & 0 & $\mid$ & 1 & 0 & 0 & 0 & 0 & 0 & 0 & 0 & 0 & 0 & 0 & 0
\end{tabular} \begin{tabular}{llllll|llllll|llllll}
0 & 0 & -1 & 0 & 0 & 0 & 0 & 0 & 1 & 0 & 0 & 0 & 0 & 0 & 0 & 0 & 0 & 0
\end{tabular} \begin{tabular}{llllll|llllll|llllll}
0 & 0 & 0 & -1 & 0 & 0 & 0 & 0 & 0 & 1 & 0 & 0 & 0 & 0 & 0 & 0 & 0 & 0
\end{tabular} \begin{tabular}{llllll|llllll|lllllll}
0 & 0 & 0 & 0 & 0 & -1 & 0 & 0 & 0 & 0 & 0 & 1 & 0 & 0 & 0 & 0 & 0 & 0
\end{tabular}
The unit motion matrix $\left[\hat{\mathbf{M}}_{\mathbf{N}}\right]_{d l, F}$ of the coupling network has been derived in section 2.4.2. This matrix contains the $d$ components of the $F$ unit actions in each of the $l$ circuits. Here, the transpose $\left[\hat{\mathbf{M}}_{\mathbf{N}}^{\mathbf{T}}\right]_{F, d l}$ is needed. To assemble all reciprocity equations, the circuit action coordinates in the vector $\left[\boldsymbol{A}_{l}\right]_{d l}$ are premultiplied by $\left[\hat{\mathbf{M}}_{\mathbf{N}}^{\mathbf{T}}\right]_{F, d l}$ to give

$$
\left[\hat{\mathbf{M}}_{\mathbf{N}}^{\mathbf{T}}\right]_{F, d l}\left[A_{l}\right]_{d l}=[\mathbf{0}]_{F}
$$

For the 2-circuit network, $F=13, d=6$, and $l=2$. Equation (11b) can, therefore, be rewritten as

$$
\left[\hat{\mathbf{M}}_{\mathbf{N}}^{\mathbf{T}}\right]_{13,12}\left[\boldsymbol{A}_{\boldsymbol{l}}\right]_{12}=[\mathbf{0}]_{13}
$$

where

$$
\begin{aligned}
& {\left[\hat{\mathbf{M}}_{\mathbf{N}}^{\mathbf{T}}\right]_{13,12}=}
\end{aligned}
$$

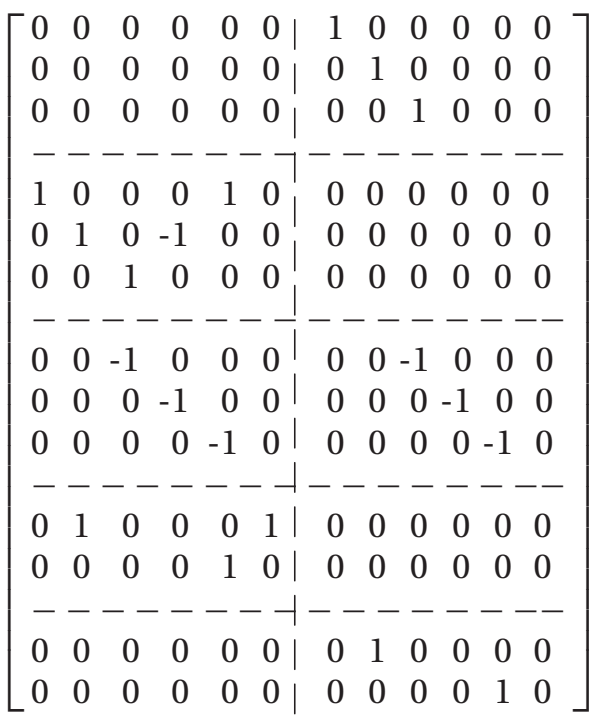


Lines partition the rows into groups of $c$ unit actions spanning the action systems of couplings $\mathbf{A}-\mathbf{E}$ in descending order. Lines partition the columns into the cutsets (and branches) $a, c$, and $d$ from left to right.
Lines partition the rows into groups of $f$ unit motions spanning the motion systems of couplings A-E in descending order. Lines partition the columns into the circuits (and chords) $b$ and $e$ from left to right.

\subsubsection{Redundant equations}

If the underconstrained coupling network is also overconstrained, the rank $a$ of $\left[\hat{\mathbf{A}}_{\mathbf{N}}^{\mathbf{T}}\right]_{C, d k}$ will be less than $C$. As noted in equation (8b), provided in the right-hand side of section 2.5.2, $C_{\mathrm{N}}=C-a$, where $C_{\mathrm{N}}$ is the nett doc.

If the $C_{\mathrm{N}}$ redundant rows are eliminated, the equation becomes

$$
\left[\hat{\mathbf{A}}_{\mathbf{N}}^{\mathbf{T}}\right]_{a, d k}\left[\hat{\mathbf{M}}_{\boldsymbol{k}}\right]_{d k}=[\mathbf{0}]_{a}
$$

It is mentioned in section 2.5.1 that the rank $a$ of $\left[\hat{\mathbf{A}}_{\mathbf{N}}^{\mathbf{T}}\right]_{17,18}$ is 15 and $C_{\mathrm{N}}$ is 2 . Rows 1 and 3 can be eliminated.
If the overconstrained coupling network is also underconstrained, the rank $m$ of $\left[\hat{\mathbf{M}}_{\mathbf{N}}^{\mathbf{T}}\right]_{F, d l}$ will be less than $F$. As noted in equation (8a), provided in the left-hand side of section 2.5.2, $F_{\mathrm{N}}=F-m$, where $F_{\mathrm{N}}$ is the nett dof.

If the $F_{\mathrm{N}}$ redundant rows are eliminated, the equation becomes

$$
\left[\hat{\mathbf{M}}_{\mathbf{N}}^{\mathbf{T}}\right]_{m, d l}\left[\boldsymbol{A}_{l}\right]_{d l}=[\mathbf{0}]_{m}
$$

It is mentioned in section 2.5.1 that the rank $m$ of $\left[\hat{\mathbf{M}}_{\mathbf{N}}^{\mathbf{T}}\right]_{13,12}$ is 10 and $F_{\mathbf{N}}$ is 3 . Rows 2,3 , and 9 can be eliminated.

\subsubsection{Virtual actions and motions}

Any real unit actions that could exist, represented by rows of $\left[\hat{\mathbf{A}}_{\mathbf{N}}^{\mathbf{T}}\right]_{C, d k}$, are absent in $\left[\hat{\mathbf{A}}_{\mathbf{N}}^{\mathbf{T}}\right]_{a, d k}$, and the rows that remain represent virtual unit actions.
Any real unit motions that could exist, represented by rows of $\left[\hat{\mathbf{M}}_{\mathbf{N}}^{\mathbf{T}}\right]_{F, d l}$, are absent in $\left[\hat{\mathbf{M}}_{\mathbf{N}}^{\mathbf{T}}\right]_{m, d l}$, and the rows that remain represent virtual unit motions.

\subsubsection{Primary variables}

Because of underconstraint the number of columns of $\left[\hat{\mathbf{A}}_{\mathbf{N}}^{\mathbf{T}}\right]_{a, d k}$ exceeds the number of rows. As noted in equation (7b), provided in the right-hand side of section 2.5.1, $F_{\mathrm{N}}=d k-a$, where $F_{\mathrm{N}}$ is the nett dof. Thus, the $d k$ unknown cutset motion components can be expressed in terms of $F_{\mathrm{N}}$ primary ones. The second, third, and eleventh terms of $\left[\boldsymbol{M}_{\boldsymbol{k}}\right]_{18}$, namely $s_{a}, t_{a}$, and $v_{c}$, are selected as primary variables.
Because of overconstraint the number of columns of $\left[\hat{\mathbf{M}}_{\mathbf{N}}^{\mathbf{T}}\right]_{m, d l}$ exceeds the number of rows. As noted in equation (7a), provided in the left-hand side of section 2.5.1, $C_{\mathrm{N}}=d l-m$, where $C_{\mathrm{N}}$ is the nett doc. Thus, the $d l$ unknown circuit motion components can be expressed in terms of $C_{\mathrm{N}}$ primary ones. The fourth and twelfth terms of $\left[\boldsymbol{A}_{\boldsymbol{l}}\right]_{12}$, namely $U_{b}$ and $W_{e}$, are selected as primary variables.

\subsection{Results}

The solution, when transposed, is

$$
\begin{aligned}
& {\left[\boldsymbol{M}_{\boldsymbol{k}}\right]_{18}^{\mathrm{T}}=\left[\left[\boldsymbol{M}_{\boldsymbol{a}}\right]_{6}^{\mathrm{T}} \vdots\left[\boldsymbol{M}_{\boldsymbol{c}}\right]_{6}^{\mathrm{T}} \vdots\left[\boldsymbol{M}_{\boldsymbol{d}}\right]_{6}^{\mathrm{T}}\right]_{18}^{\mathrm{T}}=} \\
& {\left[0, s_{a}, t_{a} ; 0,0,0 \vdots 0,0, t_{a} ; 0, v_{c}, 0 \vdots 0,0,0 ; 0, v_{c}, 0\right]^{\mathrm{T}}}
\end{aligned}
$$

The solution, when transposed, is

$$
\begin{aligned}
{\left[\boldsymbol{A}_{l}\right]_{12}^{\mathrm{T}}=} & {\left[\left[\boldsymbol{A}_{\boldsymbol{b}}\right]_{6}^{\mathrm{T}} \vdots\left[\boldsymbol{A}_{\boldsymbol{e}}\right]_{6}^{\mathrm{T}}\right]_{12}^{\mathrm{T}}=} \\
& {\left[0, U_{b}, 0 ; U_{b}, 0,-U_{b} \vdots 0,0,0 ;-U_{b}, 0, W_{e}\right]^{\mathrm{T}} }
\end{aligned}
$$




\subsubsection{Results in matrix form}

Note that equation (13a) provides the components of cutset motion systems, not those of couplings, except for the couplings represented by branches of $G_{\mathrm{C}}$. To obtain the components of motion systems for all couplings, the $d k$ components of $\left[\boldsymbol{M}_{\mathbf{k}}\right]_{d k}$, now expressed in terms of $F_{\mathrm{N}}$ primary variables, can be reassembled first in matrix form as $\left[\mathbf{M}_{\mathbf{k}}\right]_{d, k}$ and then be postmultiplied by $[\mathbf{Q}]_{k, e}$, the cutset matrix of the coupling graph $G_{C}$, to give

$[\mathbf{M}]_{d, e}=\left[\mathbf{M}_{\mathbf{k}}\right]_{d, k}[\mathbf{Q}]_{k, e}$

where $[\mathbf{M}]_{d, e}$ provides a column of $d$ components for each of the $e$ couplings. This is the same matrix as the one produced by using the adaptation of Kirchhoff's circuit law.

To obtain the components of the motion systems of all five directly coupled pairs of bodies in the 2-circuit network, equation (14a) becomes

$$
[\mathbf{M}]_{6,5}=\left[\mathbf{M}_{\mathbf{k}}\right]_{6,3}[\mathbf{Q}]_{3,5}
$$

$\left[\mathbf{M}_{\mathbf{k}}\right]_{6,3}$, derived from the vector $\left[\boldsymbol{M}_{\boldsymbol{k}}\right]_{18}$, is

$$
\left[\begin{array}{ccc}
0 & 0 & 0 \\
s_{a} & 0 & 0 \\
t_{a} & t_{a} & 0 \\
0 & 0 & 0 \\
0 & v_{c} & v_{c} \\
0 & 0 & 0
\end{array}\right]
$$

and $[\mathbf{Q}]_{3,5}$, provided by equation (3b) in section 2.3.1, is

$$
\left[\begin{array}{rrrrr}
1 & 0 & 0 & 0 & -1 \\
0 & 1 & 1 & 0 & 1 \\
0 & -1 & 0 & 1 & 0
\end{array}\right] .
$$

Hence

$$
[\mathbf{M}]_{6,5}=\left[\begin{array}{ccccc}
0 & 0 & 0 & 0 & 0 \\
s_{a} & 0 & 0 & 0 & -s_{a} \\
t_{a} & t_{a} & t_{a} & 0 & 0 \\
0 & 0 & 0 & 0 & 0 \\
0 & 0 & v_{c} & v_{c} & v_{c} \\
0 & 0 & 0 & 0 & 0
\end{array}\right]
$$

The matrices labelled equations (15a) and (15b) are identical to those previously derived in section 2.6.2.

\subsubsection{Circuit action systems}

Whereas the motions are easily recognized, the actions are not. The two circuits are shown in Figs 4 and 5 and representations of the circuit action systems are superimposed on those
Note that equation (13b) provides the components of circuit action systems, not those of couplings, except for the couplings represented by chords of $G_{\mathrm{C}}$. To obtain the components of action systems for all couplings, the $d l$ components of $\left[A_{l}\right]_{d l}$, now expressed in terms of $C_{\mathrm{N}}$ primary variables, can be reassembled first in matrix form as $\left[\mathbf{A}_{\mathbf{l}}\right]_{d, l}$ and then be postmultiplied by $[\mathbf{B}]_{l, e}$, the circuit matrix of the coupling graph $G_{\mathrm{C}}$, to give

$$
[\mathbf{A}]_{d, e}=\left[\mathbf{A}_{\mathbf{I}}\right]_{d, l}[\mathbf{B}]_{l, e}
$$

where $[\mathbf{A}]_{d, e}$ provides a column of $d$ components for each of the $e$ couplings. This is the same matrix as the one produced by using the adaptation of Kirchhoff's cutset law.

To obtain the components of the action systems for all five direct couplings in the 2-circuit network, equation (14b) becomes

$$
[\mathbf{A}]_{6,5}=\left[\mathbf{A}_{\mathbf{1}}\right]_{6,2}[\mathbf{B}]_{2,5}
$$

$\left[\mathbf{A}_{\mathbf{l}}\right]_{6,2}$, derived from the vector $\left[\boldsymbol{A}_{\boldsymbol{l}}\right]_{12}$, is

$$
\left[\begin{array}{cc}
0 & 0 \\
U_{b} & 0 \\
0 & 0 \\
U_{b} & -U_{b} \\
0 & 0 \\
-U_{b} & W_{e}
\end{array}\right]
$$

and $[\mathbf{B}]_{2,5}$, provided by equation (3a) in section 2.3.1, is

$$
\left[\begin{array}{lllll}
0 & 1 & -1 & 1 & 0 \\
1 & 0 & -1 & 0 & 1
\end{array}\right] .
$$

Hence

$$
[\mathbf{A}]_{6,5}=\left[\begin{array}{ccccc}
0 & 0 & 0 & 0 & 0 \\
0 & U_{b} & -U_{b} & U_{b} & 0 \\
0 & 0 & 0 & 0 & 0 \\
-U_{b} & U_{b} & 0 & U_{b} & -U_{b} \\
0 & 0 & 0 & 0 & 0 \\
W_{e} & -U_{b} & W_{c} & -U_{b} & W_{e}
\end{array}\right]
$$

figures. Branch $c$ of Fig. 2 belongs to both circuits. Because branch $c$ has a positive sense that is opposed to the direction in which circuits $b$ and $e$ are traversed in their positive sense, the action system for planar coupling $\mathbf{C}$ is the negative value of the sum of the circuit action systems. This action system for coupling $\mathbf{C}$ comprises the forces with lines of action that are parallel to the $z$-axis and in the plane $y=0$. 


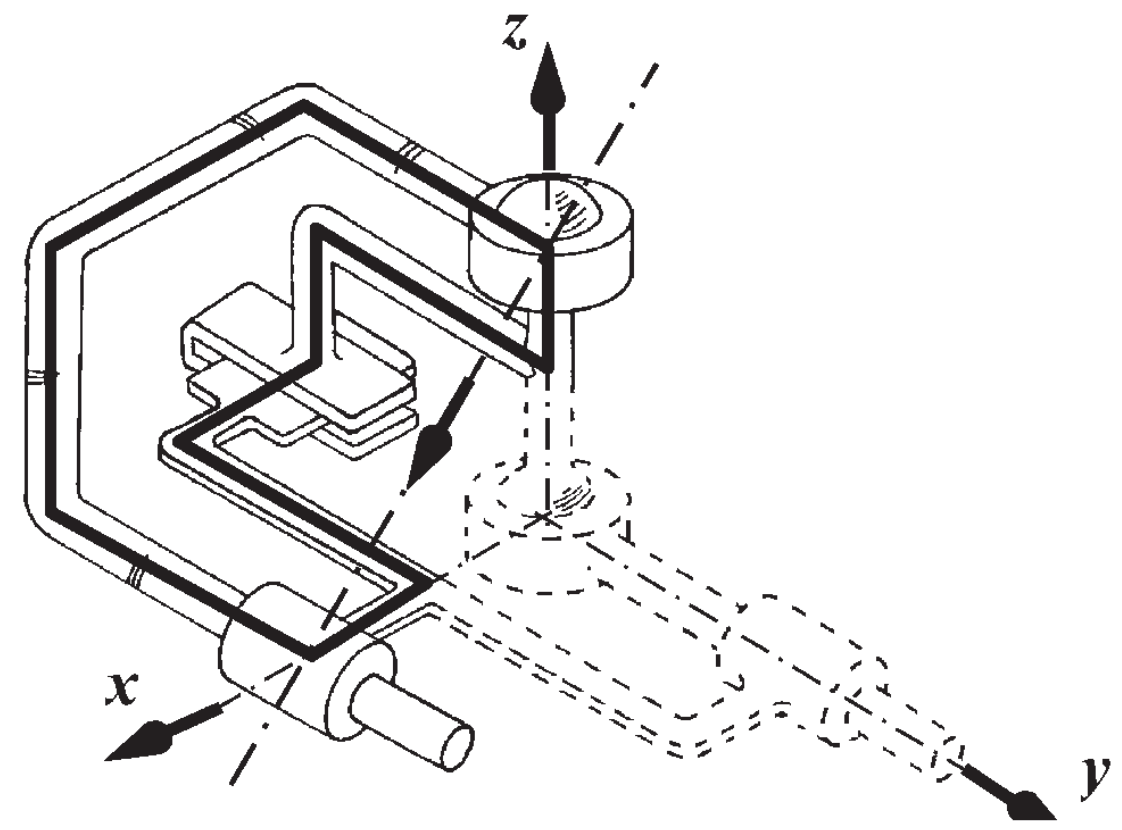

Fig. 4 Circuit $b$ and the 1-system of actions that can be locked into it: this system comprises a single force through $(0,0,1)$ and $(1,0,0)$; additionally, the $x$-component $U_{e}$ of this force is constrained to be identical to the $x$-component of the force of the 2-system associated with circuit $e$ shown in Fig. 5

\section{DISCUSSION}

\subsection{Summary of results}

Table 1 shows that, with the exception of bodies 1 and 3 , the dof of each pair of bodies is reduced by constraints provided by couplings in the coupling network other than the direct coupling that constrains them. The higher value $f$ is a characteristic of the direct coupling, whereas the lower value $f_{i j}$, the nett dof of bodies $i$ and $j$ directly coupled by that coupling, is a characteristic of the coupling

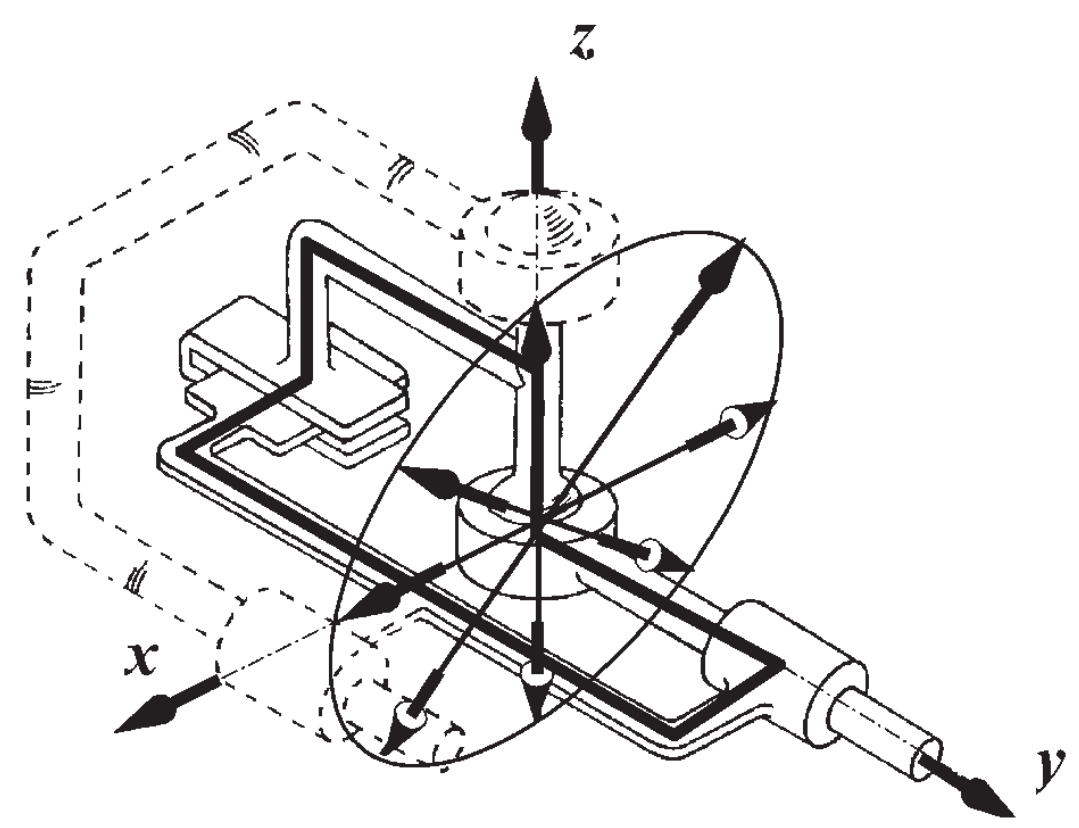

Fig. 5 Circuit $e$ and the 2-system of actions that can be locked into it: this system comprises forces with $\infty^{1}$ lines of action that belong to the pencil of lines in the plane $y=0$ having a centre at the origin 
Table 1 Summary of results for the 2-circuit network shown in Fig. 1

\begin{tabular}{|c|c|c|c|c|c|c|c|}
\hline \multirow[b]{2}{*}{$\begin{array}{l}\text { Pairs of } \\
\text { bodies }\end{array}$} & \multirow[b]{2}{*}{$\begin{array}{l}\text { Label of direct } \\
\text { coupling }\end{array}$} & \multicolumn{3}{|c|}{ Motion analysis } & \multicolumn{3}{|c|}{ Action analysis } \\
\hline & & $\begin{array}{l}f \text { for direct } \\
\text { coupling }\end{array}$ & $f_{i j}$ & $\begin{array}{l}\text { Primary } \\
\text { variables }\end{array}$ & $\begin{array}{l}c \text { for direct } \\
\text { coupling }\end{array}$ & $c_{i j}$ & $\begin{array}{l}\text { Primary } \\
\text { variables }\end{array}$ \\
\hline 1,2 & $\mathrm{D}$ & 2 & 1 & $v_{c}$ & 4 & 1 & $U_{b}$ \\
\hline 1,3 & $\mathrm{E}$ & 2 & 2 & $s_{a}, v_{c}$ & 4 & 2 & $U_{b}, W_{e}$ \\
\hline 1,4 & $\mathrm{C}$ & 3 & 2 & $t_{a}, v_{c}$ & 3 & 2 & $U_{b}, W_{e}$ \\
\hline 2,3 & Absent & $6^{\mathrm{a}}$ & 1 & $s_{a}$ & 0 & 0 & - \\
\hline 2,4 & $\mathrm{~B}$ & 3 & 1 & $t_{a}$ & 3 & 1 & $U_{b}$ \\
\hline 3,4 & A & 3 & 2 & $s_{a}, v_{c}$ & 3 & 2 & $U_{b}, W_{e}$ \\
\hline
\end{tabular}

a The absence of a direct coupling can be interpreted as a null coupling having dof of $f=6$.

network as a whole. Likewise, where a direct coupling exists, the gross doc $c$ characteristic of the direct coupling is reduced to $c_{i j}$ by the freedom permitted by other couplings. Note that, although $f+c=d$, which is 6 for this example, there is no relationship linking $f_{i j}$ and $c_{i j}$.

\subsection{Formulae for $F_{\mathrm{N}}$ and $C_{\mathrm{N}}$}

Many formulae have been suggested for $F_{\mathrm{N}}$ that are based on a count of the number of bodies and $F$, the gross dof permitted by couplings. None is universally applicable. If the coupling network is overconstrained, as well as being underconstrained, then such a formula for $F_{\mathrm{N}}$ may produce a negative integer. That is an impossible result.

In section 2.5, two formulae are derived for $F_{\mathrm{N}}$ and, dually, two more formulae for $C_{\mathrm{N}}$, the nett doc. Like $F_{\mathrm{N}}, C_{\mathrm{N}}$ must be either zero or a positive integer. The four formulae are reproduced below in tandem as dual pairs

$$
\begin{aligned}
& F_{\mathrm{N}}=F-m \\
& C_{\mathrm{N}}=C-a \\
& C_{\mathrm{N}}=d l-m \\
& F_{\mathrm{N}}=d k-a
\end{aligned}
$$

All four formulae require the determination of the rank of a matrix, either $a$ or $m$. These ranks can be eliminated to provide two more dual equations.

$$
\begin{aligned}
& C_{\mathrm{N}}-F_{\mathrm{N}}=C-d k \\
& F_{\mathrm{N}}-C_{\mathrm{N}}=F-d l
\end{aligned}
$$

Except for the change in notation, equation (16b) is reported in reference [7]. These formulae are most relevant to machinery wherein bearings of low freedom $(f=1)$ and high constraint $(c=5)$ are the most frequently used couplings. Consequently, for machinery, it is reasonable to claim that $F<C$ and it is easily shown that $l<k$, suggesting that equation (16b) is easier to use than equation (16a).

Motions are easier to recognize, and therefore easier to imagine, than internal actions. $F_{\mathrm{N}}$ is easily found by observation or model making but $C_{\mathrm{N}}$ is not. For redundant structures, where $F_{\mathrm{N}}$ is obviously zero, equation (16b) is particularly easy to use.

\subsection{The subsets of coupling networks}

The values of $C_{\mathrm{N}}$ and $F_{\mathrm{N}}$, either zero or positive, partition the set of all coupling networks into four subsets, has shown in Table 2.

\subsection{Which are the better methods?}

Two methods are described for motion analysis within underconstrained coupling networks and two methods for action analysis within overconstrained coupling networks. It is now possible to decide which of each pair of methods for the same task is the superior one.

The most time consuming, and difficult, part of the procedures is assembling the matrices $\left[\hat{\mathbf{M}}_{\mathbf{N}}\right]_{d l, F}$ and $\left[\hat{\mathbf{A}}_{\mathbf{N}}\right]_{d k, C}$ or their transpose. A measure of difficulty is the size of these matrices. They contain $d l F$ and $d k C$ elements, respectively. It is argued earlier that,

Table 2 The four subsets of coupling networks

\begin{tabular}{cccc}
\hline$F_{\mathrm{N}}$ & $C_{\mathrm{N}}$ & Alternative descriptions & Comments \\
\hline 0 & 0 & $\begin{array}{l}\text { Simply stiff/statically determinate structures } \\
\text { Redundant/statically indeterminate/ } \\
\text { overconstrained structures } \\
\text { Kinematically designed kinematic chains }\end{array}$ & $\begin{array}{c}\text { Rare: most structures are overconstrained } \\
\text { Rare: most overconstrained structures are not } \\
\text { coupling networks, they are bolted or welded } \\
\text { Rare: confined mainly to lightly loaded } \\
\text { assemblies found in instruments } \\
\text { Commonplace: most machines are based on } \\
\text { anese }\end{array}$ \\
\hline
\end{tabular}


for machinery, we can expect $F<C$ and $l<k$. Consequently, $\left[\hat{\mathbf{M}}_{\mathbf{N}}\right]_{d l, F}$ is easier to assemble than $\left[\hat{\mathbf{A}}_{\mathbf{N}}\right]_{d k, C}$. As well as there being a predominance of couplings in machinery for which $f<c$, it is suggested that the task of identifying $f$ motions that span the motion system of a coupling is easier than identifying $c$ actions that span the action system.

The evidence provided earlier suggests that the adaptation of Kirchhoff's circuit law is the preferred method for motion analysis and the method using virtual motion and virtual work is the preferred method for action analysis. This is borne out by the 2-circuit network.

\subsection{Statics}

The methods described have been applied to a study of the kinematics and statics of a two-stage epicyclic gear train [14]. Although the correct result was obtained, it became clear that a statics problem* was not a suitable example of an application of the methods described here. In statics, unlike kinematics, the coupling network is regarded as an open system on which external actions are applied. In order to use the methods described here for a statics problem, it is necessary to internalize external actions. Essentially, this means treating active couplings [2] (engines, gravitation, motors, muscles, rams) as if they are passive couplings. Alternatively, such active couplings can be thought of as being replaced by equivalent passive couplings [14] to create an overconstrained coupling network.

A difficulty arises now if, as is usual, the coupling network is already overconstrained before the external actions are internalized. The method cannot distinguish between actions that are attributable to the original overconstraint and those that are internalized in this way. Therefore, relationships between all actions that could exist are provided even though some results are not required. One way of circumventing this difficulty is to assume the original overconstraint does not exist. This was done for the gear train example: an action space of dimension $d=2$ was used wherein all forces are coplanar and parallel.

\subsection{Static and kinematic indeterminacy}

It might appear remarkable that the two methods that have been described for the analysis of actions only work for coupling networks that are statically indeterminate. This is not so remarkable, however, if it is recognized that all underconstrained coupling

\footnotetext{
*The example was chosen as a consequence of a challenge issued by a visitor who asked whether statics problems could be solved by the methods explained in this article.
}

networks are kinematically indeterminate. Many engineers have been introduced to kinematics through the medium of velocity diagrams. If a numerical answer is required for an $F_{\mathrm{N}}=1$ mechanism it is essential that the magnitude of one motion is provided. Without this information analysis can only result in the ratios of motion magnitudes. It is the same for overconstrained networks. In order to measure all actions within an overconstrained coupling network having a nett doc $C_{\mathrm{N}}$ of 1 , it is necessary to have one member instrumented, perhaps with strain gauges, so that the magnitude of one action is known. Without such numerical information, only the ratios of action magnitudes can be found.

\section{CONCLUSIONS}

1. Kirchhoff's cutset law is adapted for a study of internal actions that can result from overconstraint.

2. The concepts of cutset motions and internal virtual actions are introduced and used in a study of motions that can result from underconstraint.

3. The four methods described appear to be robust and universally applicable.

4. Of the two methods for the analysis of motions within an underconstrained coupling network, the adaptation of Kirchhoff's circulation law is the simpler one to use.

5. Of the two methods for the analysis of actions within an overconstrained coupling network, the virtual power method described is the simpler one to use.

6. Cutset (or branch) motion systems concisely represent all possible motions and circuit (or chord) action systems concisely represent all possible actions associated with coupling networks.

7. The positive senses arbitrarily assigned by the user to the edges, circuits, and cutsets of the motion and action graphs provide a quick reference to a customized sign convention.

8. Mechanical circuit theory, or kinestatics, the study of motions and actions within coupling networks in equilibrium, is an order of six times more complex than DC electrical circuit theory.

9. The 2-circuit network, being highly artificial, grossly understates the difficulties that are encountered in using the methods described.

10. Further demonstrations are provided that actions and motions are geometrically identical. The only difference is the units involved.

11. Motion can be seen; action has to be felt to be experienced. Actions within machinery attributable to overconstraint are, therefore, more difficult to recognize and imagine than motions attributable to underconstraint. 
12. Whereas $c, f, C$, and $F$ are characteristics of couplings; $c_{\mathrm{ij}}, f_{\mathrm{ij}}, C_{\mathrm{N}}$, and $F_{\mathrm{N}}$ are dependent also of the topology of their interconnections and of the geometry of their relative locations and orientation.

13. Formulae for $C_{\mathrm{N}}$ and $F_{\mathrm{N}}$ are provided, which involve the rank of a matrix. Two equations that do not involve rank are derived and the recommended one is

$$
F_{\mathrm{N}}-C_{\mathrm{N}}=F-d l
$$

\section{REFERENCES}

1 Davies, T. H. Kirchhoff's circulation law applied to multi-loop kinematic chains. Mech. Mach. Theory, 1981, 16(3), 171-183.

2 Davies, T. H. Couplings, coupling networks and their graphs. Mech. Mach. Theory, 1995, 30(7), 991-1000.

3 Tischler, C. R. and Samuel, A. E. Predicting the slop of series/in-parallel manipulators caused by joint clearances. In Sixth International Symposium on Advances in robot kinematics: analysis and control, 1998, pp. 227-236 (Kluwer Academic Press, Dordrecht).

4 Tischler, C. R. Alternative structures for robot hands. PhD Thesis, The University of Melbourne, Australia, November 1995.

5 Campos, A., Martins, D., and Guenther, R. A unified approach to differential kinematics of nonredundant manipulators. In Proceedings of the 11th International Conference on Advanced robotics, ICAR 2003, Coimbra, Portugal, 2003, Vol. 3, pp. 1837-1842 (University of Coimbra, Computer and Electrical Engineering Department).

6 Campos, A., Martins, D., and Guenther, R. Differential kinematics of robot manipulators using virtual chains. In Proceedings of the Conference on Mechatronics and robotics 2004, MechRob2004, Aachen, Germany, 2004, Vol. 3, pp. 960-965 (APS - European Centre of Mechatronics, Aachen).

7 Davies, T. H. Mechanical networks-III: wrenches on circuit screws. Mech. Mach. Theory, 1983, 18(2), 107-112.

8 Fayet, M. Distribution of wrench-twist duality in overconstrained mechanisms. J. Mech. Des., 2003, 125(1), 81-91.

9 Fayet, M. Méchanismes multiboucles I - détermination des espaces de torseurs cinématique dans un méchanismes. Mech. Mach. Theory, 1995, 30(2), 201-217.

10 Whitney, D. E. Mechanical assemblies: their design, manufacture, and role in product development, 2004 (Oxford University Press, New York, Oxford).

11 Hunt, K. H. Kinematic geometry of mechanisms, 1990, The Oxford Engineering Science Series, 7 (Oxford University Press, Oxford).

12 Chen, W.-K. Linear networks and systems, 1983 (Brooks/Cole Engineering Division, Monterey, California).

13 Baker, J. E. On relative freedom between links in kinematic chains with cross-jointing. Mech. Mach. Theory, 1980, 15(5), 397-413.

14 Davies, T. H. The 1887 committee meets again. Subject: freedom and constraint. In Proceedings of A Symposium Commemorating the Legacy, Works, and Life of Sir Robert Stawell Ball upon the 100th Anniversary of $A$ treatise on the theory of screws, University of Cambridge, Trinity College, 9-12 July 2000, available from <http://helix.gatech.edu/ball2000/CD/Papers/ ball2000-04.pdf $>$, accessed 16 March 2005.

15 Phillips, J. Freedom in machinery: Vol. 1 Introducing screw theory, 1984 (Cambridge University Press, Cambridge).

16 Phillips, J. Freedom in machinery: Vol. 2 screw theory exemplified, 1990 (Cambridge University Press, Cambridge).

\section{APPENDIX 1}

\section{Notation}

$a$

$[\mathbf{A}]_{d, C}$

$[\mathbf{A}]_{d, e}$

$\left[\boldsymbol{A}_{\boldsymbol{b}}\right]_{d}$

$\left[\mathbf{A}_{\mathbf{D}}\right]_{d, C},\left[\hat{\mathbf{A}}_{\mathbf{D}}\right]_{d, C}$

$\left[A_{l}\right]_{d l}$

$\left[\mathbf{A}_{\mathbf{1}}\right]_{d, l}$

$\left[\hat{\mathbf{A}}_{\mathbf{N}}\right]_{d k, C}$

$[\mathbf{B}]_{l, e}$

$\left[\mathbf{B}_{\mathbf{i}}\right]_{F, F}$,

$i=1,2, \ldots, l$

$\left[\mathbf{B}_{\mathbf{M}}\right]_{l, F}$

$c$

$c_{i j}$

C the rank of the network unit action matrix

action matrix of a coupling network in terms of $C_{\mathrm{N}}$ primary variables a condensed version of $[\mathbf{A}]_{d, C}$ with one column for each coupling $d$ action system components for circuit $b$ (similarly for circuit $e$ ) action and unit action matrices of the direct couplings of a coupling network

$d l$ action system

components for all $l$ circuits circuit action matrix network unit action matrix of a coupling network circuit matrix of the coupling graph $G_{\mathrm{C}}$ diagonal matrices with diagonal elements corresponding to row $i$ of $\left[\mathbf{B}_{\mathbf{M}}\right]_{l, F}$ : in practice, identification is by circuit (chord) label, e.g. $\left[\mathbf{B}_{\mathbf{b}}\right]_{F, F}$ for circuit $b$ circuit matrix of motion graph $G_{\mathrm{M}}$ the gross degree of constraint (doc) of a direct coupling

the nett (doc) of the direct coupling between bodies $i$ and $j$

the gross doc of a coupling network $=\Sigma c$ 


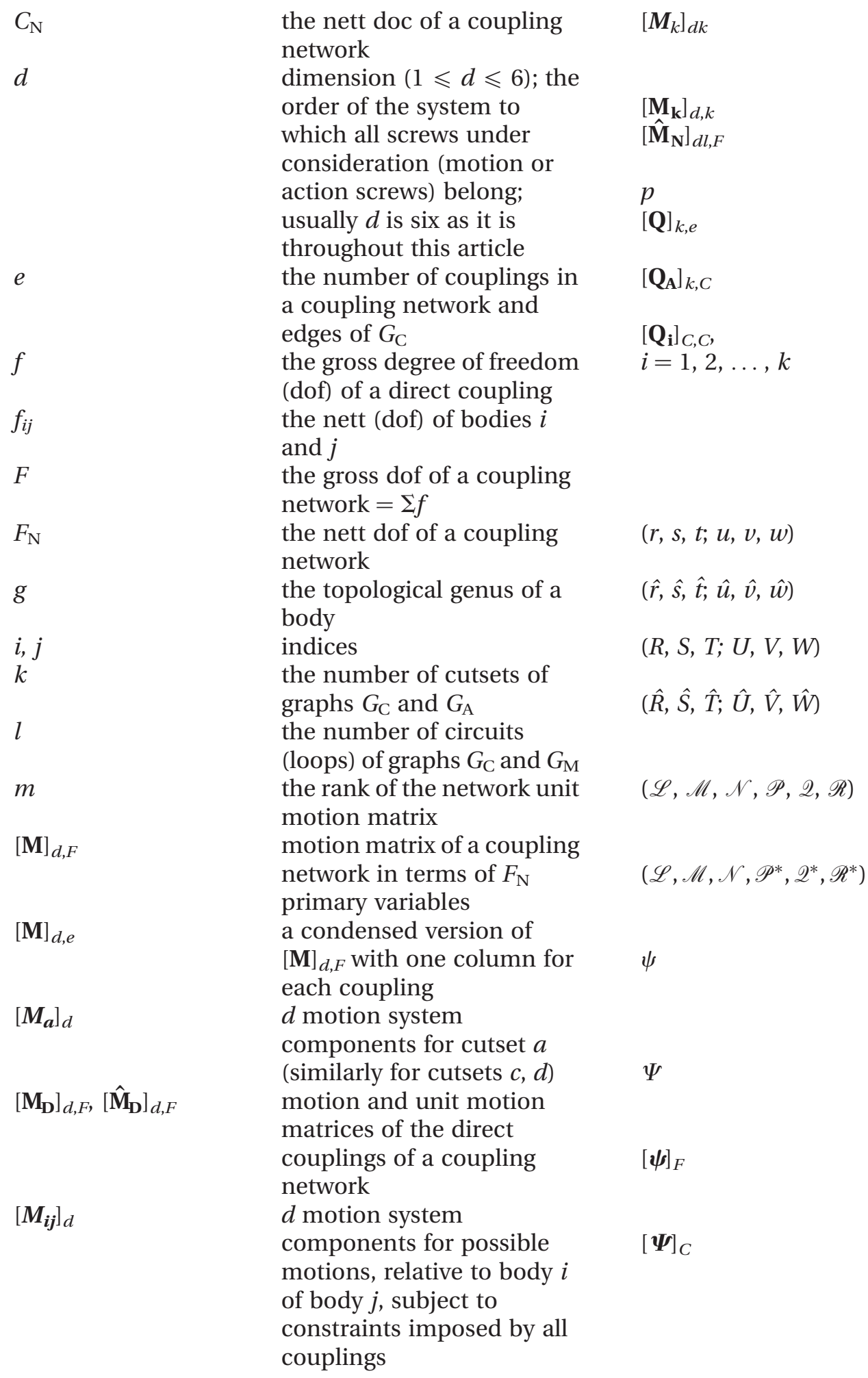

$d k$ motion system components for all $k$ cutsets cutset motion matrix network unit motion matrix of a coupling network the pitch of a screw cutset matrix of the coupling graph $G_{\mathrm{C}}$ cutset matrix of action graph $G_{\mathrm{A}}$ diagonal matrices with diagonal elements corresponding to row $i$ of $\left[\mathbf{Q}_{\mathbf{A}}\right]_{k, C}$ : in practice, identification is by cutset (branch) label, e.g. $\left[\mathbf{Q}_{\mathbf{a}}\right]_{F, F}$ for cutset $a$ motion coordinates in axis formation unit motion coordinates in axis formation action coordinates in ray formation unit action coordinates in ray formation

Plücker's line coordinates in axis formation using Hunt's notation Plücker's screw coordinates in axis formation using Hunt's notation a generalized coefficient that can be the magnitude of angular velocity or translational velocity a generalized coefficient that can be the magnitude of force or torque $F$ generalized motion magnitudes (angular or translational velocity) $C$ generalized action magnitudes (force or torque)

\section{APPENDIX 2}

The following is an outline of screw theory sufficient for an understanding of the notation. Phillips [15, 16] provides a comprehensive account of the subject but, like others, uses a different notation. Justification for the notation used here is given at the end of this section. 


\section{The screw}

As in references $[\mathbf{1 3}, \mathbf{1 5}, \mathbf{1 6}]$, the term motion is used here as a generic term for the first time derivative of the displacement of one rigid body relative to another. Thus, motion can be angular velocity, translational velocity, and the combination of the two, a twist rate about a screw.
As in references $[\mathbf{1 3}, \mathbf{1 5}, \mathbf{1 6}]$, the term action is used here as a generic term for all interactions between bodies. Thus, action can be force, torque, and the combination of the two, a wrench on a screw.

The feature common to these concepts is the screw. Much can be said about a unit screw regardless of the magnitude and units of any scalar by which it is subsequently multiplied. The unit screw is a purely geometric concept. It has an ISA and a pitch $p$. To quantify the unit screw and thereby identify its location in space relative to other screws, a global origin and orthogonal axes are necessary. The unit screw can be fully quantified by a unit sliding vector on the ISA and a 'moment' vector at the origin having dimensions of length.

The term 'moment' is wrapped in parentheses because it is borrowed from mechanics, as will become evident shortly. This geometric 'moment' vector is the resultant of the vector addition of two vectors. One of these vectors is the 'moment' at the origin of the unit sliding vector on the ISA. Like the moment of a force, it is a crossproduct of two vectors but, for the unit screw, one of them is the unit vector on a line instead of a force vector on that line. The other vector, like the moment vector of a couple, is a free vector of magnitude $p$ having the same direction as the ISA.

\section{Motion and action coordinates}

The sequence in which the two vectors are written is of critical importance.

\section{Axis formation}

When presented in axis formation, the unit screw comprises, first, the sliding unit vector on the ISA and, second, the 'moment' at the origin.

To represent motion, the unit sliding vector on the ISA is represented by $(\hat{r}, \hat{s}, \hat{t})$ and the 'moment' by $(\hat{u}, \hat{v}, \hat{w})$. The unit screw is multiplied by a generalized motion scalar $\psi$ to provide, in axis formation

$$
\psi(\hat{r}, \hat{s}, \hat{t} ; \hat{u}, \hat{v}, \hat{w})
$$

Provided that $\hat{r}, \hat{s}$, and $\hat{t}$ are not all zero, the multiplier $\psi$ has angular velocity $\left(\mathrm{T}^{-1}\right)$ units. When multiplied by $\psi$, the sliding unit vector becomes an angular velocity vector and the 'moment' at the origin becomes the velocity $(\mathrm{L} / \mathrm{T})$ of the point on the moving body located at the origin. A special case arises when $\hat{r}=\hat{s}=\hat{t}=0$. Then, the pitch $p$ is infinite, the multiplier $\psi$ has velocity (L/T) units, and only a free vector representing translational velocity remains.

\section{Ray formation}

When presented in ray formation, the unit screw comprises, first, the 'moment' at the origin and, second, the sliding unit vector on the ISA.

To represent action the 'moment' is represented by $(\hat{R}, \hat{S}, \hat{T})$ and the sliding unit vector on the ISA by $(\hat{U}, \hat{V}, \hat{W})$. The unit screw is multiplied by a generalized action scalar $\Psi$ to provide, in ray formation

$$
\Psi(\hat{R}, \hat{S}, \hat{T} ; \hat{U}, \hat{V}, \hat{W})
$$

Provided that $\hat{U}, \hat{V}$, and $\hat{W}$, are not all zero, the multiplier $\Psi$ has force (F) units. When multiplied by $\Psi$, the sliding unit vector becomes a force vector and the 'moment' at the origin becomes a moment vector (FL) at the origin, which is now a real moment of a force. A special case arises when $\hat{U}=\hat{V}=\hat{W}=0$. Then, the pitch $p$ is infinite, the multiplier $\Psi$ has moment (FL) units, and only a free vector representing torque (i.e. a couple) remains.

\section{Hunt's notation}

Plücker proposed the representation of a sliding vector on a line by six coordinates. The vector could represent a line segment, angular velocity, or force. The notation used by Hunt $[\mathbf{1 1}]$, and also by Phillips $[\mathbf{1 5}, \mathbf{1 6}]$, is $(\mathscr{L}, \mathscr{M}$, 
$\mathscr{N} ; \mathscr{P}, \mathscr{Q}, \mathscr{R}$ ), where the sum of the squares of the first three coordinates is the magnitude squared and the last three coordinates are the components of the moment or velocity at the origin. For Plücker's adaptation of line coordinates for screws, Hunt adds an asterisk as a superscript for the last three coordinates.

These six screw coordinates are identical to the motion coordinates used in this article. They would be identical also to the action coordinates as well, if those action coordinates were rewritten in axis formation. Of particular importance in section 4 is the reciprocity condition; the condition that an action screw expends no power on a motion screw. Using Hunt's notation (p. 373 of reference [11]), the reciprocity condition for two screws subscripted by numbers 1 and 2 is expressed as

$$
\mathscr{L}_{1} \mathscr{P}_{2}^{*}+\mathscr{M}_{1} \mathscr{2}_{2}^{*}+\mathscr{N}_{1} \mathscr{R}_{2}^{*}+\mathscr{L}_{2} \mathscr{P}_{1}^{*}+\mathscr{M}_{2} \mathscr{Q}_{1}^{*}+\mathscr{N}_{2} \mathscr{R}_{1}^{*}=0
$$

Using the notation used in this article, the same condition is expressed as

$$
r R+s S+t T+u U+v V+w W=0
$$

It is suggested that the latter has several advantages: it is sufficient to remember the consecutive sequence $r-w$; neither superscripted asterisks nor subscripts are needed; $T$ is appropriate for torque about the $z$-axis and $u, v$ for velocity components in the $x$ - and $y$-directions.

\section{APPENDIX 3}

\section{Unit motion screw coordinates}

For a coupling with a gross $\operatorname{dof} f>1$, there are an infinite number of choices of $f$ independent screws that together span the $f$-system of motion screws associated with the coupling. The 2-circuit network used as an example in this article is specially designed so that, by making suitable choices, all unit screw coordinates are zero, 1 or -1 . The chosen unit screw coordinates, provided in Table 3, are required in section 2.4.1 for $\left[\hat{\mathbf{M}}_{\mathbf{D}}\right]_{6,13}$, the unit motion matrix for direct couplings, and the 13 magnitudes are required for $[\boldsymbol{\psi}]_{13}$.

\section{Unit action screw coordinates}

The screw coordinates for these actions, provided in Table 4, are required in section 2.4.1 for $\left[\hat{\mathbf{A}}_{\mathbf{D}}\right]_{6,17}$, the unit action matrix for direct couplings, and the 17 magnitudes are required for $[\boldsymbol{\Psi}]_{17}$.

\begin{tabular}{|c|c|c|c|c|}
\hline \multirow[b]{2}{*}{ Coupling label, type, $f$, location } & \multirow[b]{2}{*}{$\begin{array}{l}\text { Rotational or translational } \\
\text { velocity (direction) }\end{array}$} & \multirow[b]{2}{*}{ Magnitude } & \multicolumn{2}{|c|}{$\begin{array}{l}\text { Unit motion screw coordinates } \\
\text { in axis formation }\end{array}$} \\
\hline & & & $\begin{array}{l}\text { Angular } \\
\text { velocity }\end{array}$ & $\begin{array}{l}\text { Velocity of the } \\
\text { point at the origin }\end{array}$ \\
\hline \multirow[t]{3}{*}{ A, spheric, 3 , centre at $(0,0,0)$} & Rotational $(x)$ & $r_{a}$ & $1,0,0$ & $0,0,0$ \\
\hline & Rotational $(y)$ & $s_{a}$ & $0,1,0$ & $0,0,0$ \\
\hline & Rotational $(z)$ & $t_{a}$ & $0,0,1$ & $0,0,0$ \\
\hline \multirow[t]{3}{*}{$\mathrm{B}$, spheric, 3 , centre at $(0,0,1)$} & Rotational $(x)$ & $r_{b}$ & $1,0,0$ & $0,1,0$ \\
\hline & Rotational $(y)$ & $s_{b}$ & $0,1,0$ & $-1,0,0$ \\
\hline & Rotational $(z)$ & $t_{b}$ & $0,0,1$ & $0,0,0$ \\
\hline \multirow{3}{*}{$\begin{array}{l}\text { C, planar (ebene), 3, plane } \\
\text { perpendicular to the } z \text {-axis }\end{array}$} & Rotational $(z)$ & $t_{c}$ & $0,0,1$ & $0,0,0$ \\
\hline & Translational $(x)$ & $u_{c}$ & $0,0,0$ & $1,0,0$ \\
\hline & Translational $(y)$ & $v_{c}$ & $0,0,0$ & $0,1,0$ \\
\hline \multirow{2}{*}{$\begin{array}{l}\mathrm{D} \text {, cylindrical, } 2 \text {, axis through }(1,0,0) \\
\text { parallel to the } y \text {-axis }\end{array}$} & Rotational $(y)$ & $s_{d}$ & $0,1,0$ & $0,0,1$ \\
\hline & Translational $(y)$ & $v_{d}$ & $0,0,0$ & $0,1,0$ \\
\hline \multirow{2}{*}{$\begin{array}{l}\text { E, cylindrical, } 2 \text {, axis coincident with } \\
\text { the } y \text {-axis }\end{array}$} & Rotational $(y)$ & $s_{e}$ & $0,1,0$ & $0,0,0$ \\
\hline & Translational $(y)$ & $v_{e}$ & $0,0,0$ & $0,1,0$ \\
\hline
\end{tabular}

Table 3 Motions spanning the systems of motions allowed by couplings

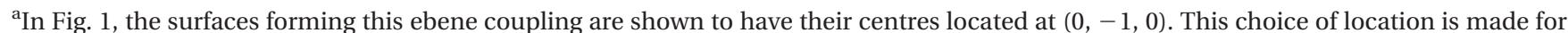
clarity. The only feature determining the 3 -system of motions that the coupling allows is the direction of the normal to the surfaces. One of the three motions chosen to span this 3-system is an angular velocity. The axis of rotation can be any line parallel to the $z$-axis. Here, but not in reference [1], the $z$-axis itself is that chosen rotation axis. 
Table 4 Actions spanning the systems of actions that can be transmitted by couplings

Unit action screw coordinates in ray formation

\begin{tabular}{|c|c|c|c|c|}
\hline Coupling label, type, $c$, location & $\begin{array}{l}\text { Force or torque } \\
\text { (direction) }\end{array}$ & Magnitude & $\begin{array}{l}\text { Moment at } \\
\text { origin }\end{array}$ & Force \\
\hline \multirow[t]{2}{*}{ A, spheric, 3 , centre at $(0,0,0)$} & Force $(x)$ & $U_{a}$ & $0, \quad 0,0$ & $1,0,0$ \\
\hline & Force $(z)$ & $W_{a}$ & $0, \quad 0,0$ & $0,0,1$ \\
\hline \multirow[t]{3}{*}{$\mathrm{B}$, spheric, 3 , centre at $(0,0,1)$} & Force $(x)$ & $U_{b}$ & $0, \quad 1,0$ & $1,0,0$ \\
\hline & Force $(y)$ & $V_{b}$ & $-1, \quad 0,0$ & $0,1,0$ \\
\hline & Force $(z)$ & $W_{b}$ & $0, \quad 0,0$ & $0,0,1$ \\
\hline \multirow{2}{*}{$\begin{array}{l}\text { C, planar (ebene), 3, plane } \\
\text { perpendicular to the } z \text {-axis }{ }^{\text {a }}\end{array}$} & Torque $(y)$ & $S_{c}$ & $0, \quad 1,0$ & $0,0,0$ \\
\hline & Force $(z)$ & $W_{c}$ & $0, \quad 0,0$ & $0,0,1$ \\
\hline \multirow{4}{*}{$\begin{array}{l}\mathrm{D} \text {, cylindrical, } 4 \text {, axis through }(1 \text {, } \\
0,0) \text {, parallel to the } y \text {-axis }\end{array}$} & Torque $(x)$ & $R_{d}$ & $1, \quad 0,0$ & $0,0,0$ \\
\hline & Torque $(z)$ & $T_{d}$ & $0, \quad 0,1$ & $0,0,0$ \\
\hline & Force $(x)$ & $U_{d}$ & $0, \quad 0,0$ & $1,0,0$ \\
\hline & Force $(z)$ & $W_{d}$ & $0,-1,0$ & $0,0,1$ \\
\hline \multirow{3}{*}{$\begin{array}{l}\text { E, cylindrical, } 4 \text {, axis coincident } \\
\text { with the } y \text {-axis }\end{array}$} & Torque $(x)$ & $R_{e}$ & $1, \quad 0,0$ & $0,0,0$ \\
\hline & Torque $(z)$ & $T_{e}$ & $0, \quad 0,1$ & $0,0,0$ \\
\hline & Force $(z)$ & $W_{e}$ & $0, \quad 0,0$ & $0,0,1$ \\
\hline
\end{tabular}

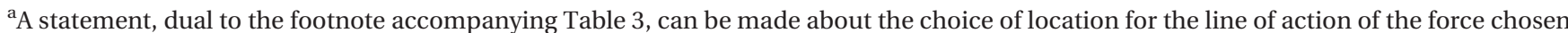
as one of the three actions spanning the action system of the ebene coupling. Here, the line of action chosen is the $z$-axis. 